March 24, 2010

Imperial-TP-2010-CH-01

MIT-CTP-4128

\title{
Background independent action for double field theory
}

\author{
Olaf Hohm¹, Chris Hull, and Barton Zwiebach ${ }^{1}$ \\ ${ }^{1}$ Center for Theoretical Physics \\ Massachusetts Institute of Technology \\ Cambridge, MA 02139, USA \\ ohohm@mit.edu,zwiebach@mit.edu \\ ${ }^{2}$ The Blackett Laboratory \\ Imperial College London \\ Prince Consort Road \\ LondonSW7@AZ,U.K. \\ c.hull@imperial.ac.uk
}

\begin{abstract}
Double field theory describes a massless subsector of closed string theory with both momentum and winding excitations. The gauge algebra is governed by the Courant bracket in certain subsectors of this double field theory. We construct the associated nonlinear backgroundindependent action that is T-duality invariant and realizes the Courant gauge algebra. The action is the sum of a standard action for gravity, antisymmetric tensor, and dilaton fields written with ordinary derivatives, a similar action for dual fields with dual derivatives, and a mixed term that is needed for gauge invariance.
\end{abstract}




\section{Contents}

1 Introduction $\quad 1$

2 Action and gauge transformations 5

2.1 Background independence . . . . . . . . . . . . . . . . 5

2.2 The gauge transformations in background independent form . . . . . . . . . . 7

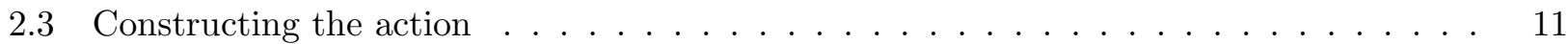

$2.4 \mathrm{O}(\mathrm{D}, \mathrm{D})$ invariance $\ldots \ldots \ldots \ldots \ldots \ldots \ldots \ldots \ldots$

2.5 The strong constraint and restricted fields . . . . . . . . . . . . 17

3 Reduction to Einstein gravity and derivative expansion 19

3.1 Reduction to the Einstein-Kalb-Ramond-dilaton action . . . . . . . . . . . . . . 19

3.2 Tilde derivative expansion, dual structure, and gauge invariance . . . . . . . . . . . 21

4 Towards an $\mathrm{O}(\mathrm{D}, \mathrm{D})$ geometry 23

$4.1 \mathrm{O}(\mathrm{D}, \mathrm{D})$ covariant derivatives and gauge transformations . . . . . . . . . . 24

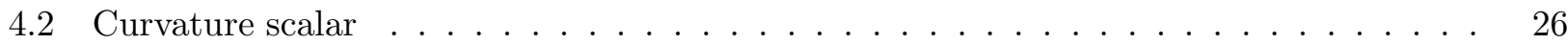

4.3 T-duality and gauge invariance . . . . . . . . . . . . . . . 30

5 Concluding remarks $\quad \lcm{32}$

A Derivation of the background independent gauge transformations 34

B Explicit check of gauge invariance $\quad 36$

\begin{tabular}{ll|} 
C Properties of the curvature scalar & 39
\end{tabular}

\section{Introduction}

Double field theory is a field theory on the doubled torus that arises when the usual toroidal coordinates $x^{i}$ are supplemented with coordinates $\tilde{x}_{i}$ associated with winding excitations. Closed string field theory in toroidal backgrounds is, by construction, a double field theory [1. In recent papers we have started the construction of a double field theory based on the massless fields of closed string theory [2, 3]. In this theory the gravity field $h_{i j}$, the antisymmetric tensor $b_{i j}$, and the dilaton $d$ all depend on the coordinates $x^{i}$ and $\tilde{x}_{i}$. The construction is novel and requires a constraint that arises from the $L_{0}-\bar{L}_{0}=0$ constraint of closed string theory: all fields and gauge parameters must be annihilated by the differential operator $\partial_{i} \tilde{\partial}^{i}$, where a sum over $i$ is understood. This 'massless' double field theory was constructed to cubic order in the fields and to this order it is gauge invariant and has a remarkable T-duality symmetry. It is an open question whether a complete nonlinear gauge-invariant extension of this massless theory exists. It would not be a conventional low-energy limit of closed string theory, and it is not yet clear whether or not this could be a consistent truncation of string theory. It would be simpler than closed string theory, however, and the ideas of doubled geometry as well as some of the essential features of closed string theory should be more accessible there. Earlier work in double field theory includes that of Tseytlin [4] and Siegel [5]. 
Rather than proceed with the full higher order construction, we will here focus on a subsector of the full double field theory satisfying a constraint that is stronger than the one discussed above and complete the construction of this subsector. The constraint from string field theory requires that all fields and gauge parameters are annihilated by $\partial_{i} \tilde{\partial}^{i}$, but the strong constraint requires that in addition all products of fields and gauge parameters are also annihilated by $\partial_{i} \tilde{\partial}^{i}$. With this strong constraint we were able 3 , to find a consistent set of gauge transformations to all orders and show that their gauge algebra is governed by the Courant bracket of generalised geometry [7, 8, 9, 10, 11]. In this paper we continue the analysis and construct the action to all orders in the fluctuating fields. We do so by constructing a background independent action. The fluctuations and the background fields of the cubic action give enough clues for the definition of full fields in terms of which the action can be neatly constructed. We use the field $\mathcal{E}_{i j}=g_{i j}+b_{i j}$, comprising of the metric $g_{i j}$ and the antisymmetric tensor $b_{i j}$, as well as a dilaton field $d$.

A striking feature of our theory is its duality symmetry. If the spacetime is the product of $n$ dimensional Minkowski space $\mathbb{R}^{n-1,1}$ and a torus $T^{d}$, string theory has $O(d, d ; \mathbb{Z})$ T-duality symmetry [12, 13] (see also [14] and references therein). In our formulation, the torus coordinates are doubled, and the double field theory has an $O(d, d ; \mathbb{Z})$ symmetry that acts naturally on the $2 d$ coordinates $(x, \tilde{x})$ of the doubled torus. It is formally useful to double the non-compact coordinates also, and our double field theory in that case has a continuous $O(n, n)$ symmetry. In particular, when formulated in non-compact $\mathbb{R}^{2 D}$, the double field theory has a continuous $O(D, D)$ symmetry. Compactifying $2 d$ of these dimensions on a double torus breaks this to $O(n, n) \times O(d, d ; \mathbb{Z})$. Restricting the fields to be independent of the extra $n$ non-compact coordinates breaks $O(n, n)$ to the Lorentz group $O(n-1,1)$. Then this $O(D, D)$ symmetry ensures the Lorentz and T-duality symmetries of the compactified cases relevant to string theory, and it will often be convenient to simply refer to the $O(D, D)$ symmetry in what follows. The constraint $\partial_{i} \tilde{\partial}^{i}=0$ is $O(D, D)$ invariant and can be written as $\partial^{M} \partial_{M}=0$ upon collecting the coordinates $x^{i}$ and dual coordinates $\tilde{x}_{i}$ into an $O(D, D)$ vector $X^{M}$ and using the constant $O(D, D)$ invariant metric $\eta_{M N}$ to raise and lower indices, so that $\partial^{M} \partial_{M}=\eta^{M N} \partial_{M} \partial_{N}$.

The strong constraint introduced above is in fact so strong that it means that there is a choice of coordinates $\left(x^{\prime}, \tilde{x}^{\prime}\right)$, related to the original coordinates by $O(D, D)$, in which the doubled fields only depend on half of the coordinates: they depend on the $x^{\prime}$ and are independent of the $\tilde{x}^{\prime}$. We refer to such fields as restricted to the null subspace with coordinates $x^{\prime}[3]$. We shall show that all solutions of the strong constraint are in fact related to a conventional field theory by $O(D, D)$ in this way.

It is interesting to compare with the work of Siegel [5], who performed a direct construction of the strongly constrained theory discussed above. The gauge algebra, which coincides with ours, was his starting point. Siegel also constructed an action, but his formalism is sufficiently different from ours that direct comparison is not straightforward. He uses vielbeins and an enlarged tangent space symmetry, while we simply use the familiar field $\mathcal{E}_{i j}$ and the dilaton. We hope that our identification and discussion of the role of the Courant bracket as well as the explicit and concrete expressions for the action given in this paper will stimulate further analysis and a detailed comparison. 
The main result in this paper is the action, which takes the form

$$
\begin{aligned}
S=\int d x d \tilde{x} e^{-2 d}[ & -\frac{1}{4} g^{i k} g^{j l} \mathcal{D}^{p} \mathcal{E}_{k l} \mathcal{D}_{p} \mathcal{E}_{i j}+\frac{1}{4} g^{k l}\left(\mathcal{D}^{j} \mathcal{E}_{i k} \mathcal{D}^{i} \mathcal{E}_{j l}+\overline{\mathcal{D}}^{j} \mathcal{E}_{k i} \overline{\mathcal{D}}^{i} \mathcal{E}_{l j}\right) \\
& \left.+\left(\mathcal{D}^{i} d \overline{\mathcal{D}}^{j} \mathcal{E}_{i j}+\overline{\mathcal{D}}^{i} d \mathcal{D}^{j} \mathcal{E}_{j i}\right)+4 \mathcal{D}^{i} d \mathcal{D}_{i} d\right] .
\end{aligned}
$$

In this action, the calligraphic derivatives are defined by

$$
\mathcal{D}_{i} \equiv \frac{\partial}{\partial x^{i}}-\mathcal{E}_{i k} \frac{\partial}{\partial \tilde{x}_{k}}, \quad \overline{\mathcal{D}}_{i} \equiv \frac{\partial}{\partial x^{i}}+\mathcal{E}_{k i} \frac{\partial}{\partial \tilde{x}_{k}},
$$

and all indices are raised with $g^{i j}$, which is the inverse of the metric $g_{i j}=\frac{1}{2}\left(\mathcal{E}_{i j}+\mathcal{E}_{j i}\right)$. The gauge transformations that leave the action invariant take the form

$$
\begin{aligned}
\delta \mathcal{E}_{i j} & =\mathcal{D}_{i} \tilde{\xi}_{j}-\overline{\mathcal{D}}_{j} \tilde{\xi}_{i}+\xi^{M} \partial_{M} \mathcal{E}_{i j}+\mathcal{D}_{i} \xi^{k} \mathcal{E}_{k j}+\overline{\mathcal{D}}_{j} \xi^{k} \mathcal{E}_{i k} \\
\delta d & =-\frac{1}{2} \partial_{M} \xi^{M}+\xi^{M} \partial_{M} d .
\end{aligned}
$$

Here $\xi^{M} \partial_{M}=\xi^{i} \partial_{i}+\tilde{\xi}_{i} \tilde{\partial}^{i}$ and $\partial_{M} \xi^{M}=\partial_{i} \xi^{i}+\tilde{\partial}^{i} \tilde{\xi}_{i}$. All fields, gauge parameters, and all possible products of them are assumed to be annihilated by $\partial^{M} \partial_{M}$. The algebra of gauge transformations is governed by an $O(D, D)$ covariant "C-bracket" [5, 3] which reduces to the Courant bracket when fields and gauge parameters are T-dual to ones that are independent of winding coordinates. This action is also invariant under the non-linearly realized $O(D, D)$ duality transformations that transform $\mathcal{E}$ projectively and leave the dilaton invariant. Using matrix notation,

$$
\mathcal{E}^{\prime}\left(X^{\prime}\right)=(a \mathcal{E}(X)+b)(c \mathcal{E}(X)+d)^{-1}, \quad d^{\prime}\left(X^{\prime}\right)=d(X), \quad X^{\prime}=h X,
$$

where

$$
h=\left(\begin{array}{ll}
a & b \\
c & d
\end{array}\right) \in O(D, D) .
$$

Finally, when fields are assumed to be independent of the $\tilde{x}$ coordinates, the action (1.1) reduces to a form that is field-redefinition equivalent to the familiar action

$$
S_{*}=\int d x \sqrt{-g} e^{-2 \phi}\left[R+4(\partial \phi)^{2}-\frac{1}{12} H^{2}\right] .
$$

In particular, the usual scalar dilaton $\phi$ is related to the field $d$ used here by $\sqrt{-g} e^{-2 \phi}=e^{-2 d}$, so that $e^{-2 d}$ is a scalar density. A by-product of our analysis is that we find the explicit form of the field redefinitions that relate the metric and $b$-field variables used in string theory to those used in the conventional Einstein plus $b$-field theory (see $₫ 2.2$ ), completing the work of Refs. [15, 2].

There has been some interest in gravity theories that replace the metric with a non-symmetric tensor field; see e.g. [16]. The field $\mathcal{E}_{i j}$ is non-symmetric, and we believe (1.1) is a natural action written in terms of this variable. After rotating to a T-duality frame in which all tilde-derivatives are zero, the action (given in (3.1)) is a rather simple rewriting of the conventional action $S_{*}$ using the $\mathcal{E}$ field variable. The theory defined by (1.1) provides therefore a reformulation of $S_{*}$ in which $O(D, D)$ is a symmetry. Moreover, in contrast to theories of non-symmetric gravity of the type discussed in [16], 
here the symmetric and antisymmetric parts of $\mathcal{E}_{i j}$ do not provide irreducible representations of the gauge and duality symmetries but rather transform into each other.

All terms in the action (1.1) contain two derivatives. It is natural to examine a tilde-derivative $(\tilde{\partial})$ expansion $S=S^{(0)}+S^{(1)}+S^{(2)}$ where $S^{(k)}$ contains $k$ tilde derivatives. The action $S^{(0)}(\mathcal{E}, d, \partial)$ is obtained by setting all terms with tilde-derivatives to zero and thus, as mentioned above, is equivalent to $S_{*}$. It is interesting that $S^{(2)}$ is in fact equal to $S^{(0)}\left(\mathcal{E}^{-1}, d, \tilde{\partial}\right)$, the conventional action for gravity, $b$-field, and dilaton but with $\mathcal{E} \rightarrow \mathcal{E}^{-1}$ and $\partial \rightarrow \tilde{\partial}$, the changes associated with inversion duality. The action $S^{(1)}$ is a curious mix, with terms that involve one derivative of each type. Gauge invariance of the action will be demonstrated explicitly using a similar tilde-derivative expansion of the gauge transformations. Specifically, the gauge transformations (1.3) can be rewritten as

$$
\begin{aligned}
\delta_{\xi} \mathcal{E}_{i j}= & \mathcal{L}_{\xi} \mathcal{E}_{i j}+\partial_{i} \tilde{\xi}_{j}-\partial_{j} \tilde{\xi}_{i} \\
& +\mathcal{L}_{\tilde{\xi}} \mathcal{E}_{i j}-\mathcal{E}_{i k}\left(\tilde{\partial}^{k} \xi^{l}-\tilde{\partial}^{l} \xi^{k}\right) \mathcal{E}_{l j} .
\end{aligned}
$$

Here, $\mathcal{L}_{\xi}$ and $\mathcal{L}_{\tilde{\xi}}$ are the Lie derivatives with respect to the parameters $\xi^{i}$ and $\tilde{\xi}_{i}$ respectively. We note that, despite appearance in (1.3), these two gauge parameters enter completely democratically as they combine naturally into the $O(D, D)$ vector $\xi^{M}=\left(\tilde{\xi}_{i}, \xi^{i}\right)$. In fact, we will show that under the inversion duality $\mathcal{E} \rightarrow \mathcal{E}^{-1}, \partial \rightarrow \tilde{\partial}$ the role of $\xi^{i}$ and $\tilde{\xi}_{i}$ in (1.7) gets precisely interchanged.

We give some preliminary discussion of an attempt to formulate an ' $O(D, D)$ geometry'. The analysis of $O(D, D)$ invariance of the action and covariance of the gauge transformations is facilitated by the introduction of $O(D, D)$ 'covariant' derivatives that map $O(D, D)$ tensors to $O(D, D)$ tensors. We also construct an $O(D, D)$-invariant scalar curvature $\mathcal{R}(\mathcal{E}, d)$, all of whose terms contain two derivatives (see eqn. (4.33)). Being a scalar means that it transforms under gauge transformations as $\delta_{\xi} \mathcal{R}=\xi^{M} \partial_{M} \mathcal{R}$. Since $e^{-2 d}$ is a density we obtain an $O(D, D)$ invariant and gauge invariant action by writing

$$
S^{\prime}=\int d x d \tilde{x} e^{-2 d} \mathcal{R}(\mathcal{E}, d)
$$

The two actions $S^{\prime}$ and $S$ in (1.1) differ by integrals of total derivatives and so are equivalent. It is a nontrivial fact that the dilaton equation of motion following from (1.8) is simply $\mathcal{R}=0$. The variations of $d$ within $\mathcal{R}$ combine to a total derivative and do not contribute to the field equation. When we set tilde-derivatives to zero we find that, after the field redefinition that trades $d$ for $\phi$, the scalar $\mathcal{R}$ becomes

$$
\left.\mathcal{R}(\mathcal{E}, d)\right|_{\tilde{\partial}=0}=R+4 \square \phi-4(\partial \phi)^{2}-\frac{1}{12} H^{2} .
$$

Thus assuming that all fields are independent of the $\tilde{x}$ coordinates the action $S^{\prime}$ (without discarding total derivatives) becomes:

$$
S_{*}^{\prime}=\int d x \sqrt{-g} e^{-2 \phi}\left[R+4 \square \phi-4(\partial \phi)^{2}-\frac{1}{12} H^{2}\right] .
$$

This action inherits the curious property of $S^{\prime}$ : the equation of motion for the dilaton is precisely the vanishing of the terms in the square brackets. Note that this is not true for the action $S_{*}$ in (1.6). The 
curvature $\mathcal{R}$ is precisely the beta function $\beta^{d}$ for the $O(D, D)$ scalar dilaton $d$. The spacetime action in [17] used a Lagrangian proportional to $\beta^{d}$. The gauge scalar $\mathcal{R}(\mathcal{E}, d)$ is also an $O(D, D)$ scalar and can be viewed as a generalization of the scalar curvature of Einstein's theory. It is interesting that the object that admits a generalization is the precise combination of terms indicated in (1.9).

We also investigate the relation between the $O(D, D)$ duality symmetry and the gauge symmetry. We show that $G L(D)$ transformations and constant shifts of the $b$-field are special gauge symmetries, but in general the remaining $O(D, D)$ transformations do not arise from gauge symmetries. However, we find that in the special case in which we truncate the theory to one with fields that are independent of $d$ coordinates and their $d$ duals there is an $O(d, d)$ symmetry that arises from gauge symmetries. This gives a geometric insight into the $O(d, d)$ symmetry [25] that emerges from Kaluza-Klein reduction on a $d$-torus.

\section{Action and gauge transformations}

In this section we start by proving background independence of the cubic action given in [2]. Then we determine the manifestly background independent form both for the action and the gauge transformations to all orders in the fluctuations. In addition, we prove the $O(D, D)$ invariance of the action and the $O(D, D)$ covariance of the gauge transformations. Finally, we show that the strong form of the constraint $\partial^{M} \partial_{M}=0$ guarantees that one can always find an $O(D, D)$ transformation that rotates to a duality frame where all fields are independent of the winding coordinates.

\subsection{Background independence}

In [2] we constructed the double field theory action for the dilaton $d$ and the field $e_{i j}$ giving the fluctuation in the metric and anti-symmetric tensor gauge field around the constant background $E_{i j}=$ $G_{i j}+B_{i j}$. The action was obtained to cubic order in the fields $e_{i j}$ and $d$ and takes the form (with $\left.2 \kappa^{2}=1\right)$ :

$$
\begin{aligned}
S= & \left.\int d x d \tilde{x}\right]\left[\frac{1}{4} e_{i j} \square e^{i j}+\frac{1}{4}\left(\bar{D}^{j} e_{i j}\right)^{2}+\frac{1}{4}\left(D^{i} e_{i j}\right)^{2}-2 d D^{i} \bar{D}^{j} e_{i j}-4 d \square d\right. \\
& +\frac{1}{4} e_{i j}\left(\left(D^{i} e_{k l}\right)\left(\bar{D}^{j} e^{k l}\right)-\left(D^{i} e_{k l}\right)\left(\bar{D}^{l} e^{k j}\right)-\left(D^{k} e^{i l}\right)\left(\bar{D}^{j} e_{k l}\right)\right) \\
& +\frac{1}{2} d\left(\left(D^{i} e_{i j}\right)^{2}+\left(\bar{D}^{j} e_{i j}\right)^{2}+\frac{1}{2}\left(D_{k} e_{i j}\right)^{2}+\frac{1}{2}\left(\bar{D}_{k} e_{i j}\right)^{2}+2 e^{i j}\left(D_{i} D^{k} e_{k j}+\bar{D}_{j} \bar{D}^{k} e_{i k}\right)\right) \\
& \left.+4 e_{i j} d D^{i} \bar{D}^{j} d+4 d^{2} \square d\right] .
\end{aligned}
$$

In 22] the corresponding gauge transformations were found to linear order in the fields and this action was shown to be invariant under these gauge transformations up to terms quadratic in the fields, provided the fields and gauge parameters are annihilated by $\partial_{i} \tilde{\partial}^{i}$. The background dependence in the action enters through the derivatives

$$
D_{i}=\frac{\partial}{\partial x^{i}}-E_{i k} \frac{\partial}{\partial \tilde{x}_{k}}, \quad \bar{D}_{i}=\frac{\partial}{\partial x^{i}}+E_{i k}^{t} \frac{\partial}{\partial \tilde{x}_{k}}
$$


and the inverse metric $G^{i j}$, which is used to raise indices in this subsection. Note that the field $e_{i j}$ and the derivatives $D_{i}$ and $\bar{D}_{i}$ are defined with indices down.

Background independence is a property that the above action should inherit from the full string theory. This means that one can absorb a constant part of the fluctuation field $e_{i j}$ into a change of the background field $E_{i j}$. The dilaton plays no role in the background dependence; we will display only the $E$ and $e$ dependence in the action and write $S\left[E_{i j}, e_{i j}\right]$. Let $\chi_{i j}$ be an infinitesimal, constant part of the field $e_{i j}$. The statement of background independence is that

$$
S\left[E_{i j}, e_{i j}+\chi_{i j}\right]=S\left[E_{i j}+\chi_{i j}, e_{i j}^{\prime}=e_{i j}+f_{i j}(\chi, e)\right]
$$

where $f(\chi, e)$ is a function that is linear in $\chi$ and to leading order linear in $e_{i j}$. This function is needed in general; it means that the new fluctuation field $e_{i j}^{\prime}$ is a field redefined version of $e_{i j}$. Note that vanishing e must imply vanishing $e^{\prime}$, for consistency. Thus, there is no $e_{i j}$-independent term in $f_{i j}$. Letting $E_{i j} \rightarrow E_{i j}-\chi_{i j}$ and noting that to leading order

$$
e_{i j}=e_{i j}^{\prime}-f_{i j}\left(\chi, e^{\prime}\right)
$$

we have

$$
S\left[E_{i j}-\chi_{i j}, e_{i j}^{\prime}+\chi_{i j}-f_{i j}\left(\chi, e^{\prime}\right)\right]=S\left[E_{i j}, e_{i j}^{\prime}\right] .
$$

Dropping the primes, the condition of background independence to leading order in $e$ becomes

$$
S\left[E_{i j}-\chi_{i j}, e_{i j}+\chi_{i j}-f_{i j}(\chi, e)\right]=S\left[E_{i j}, e_{i j}\right]+O\left(e^{3}\right) .
$$

With the action calculated to cubic order, we can only determine the leading terms in $f_{i j}$, those linear in $e$. Our check of background independence fixes $f_{i j}$ to be

$$
f_{i j}(\chi, e)=\frac{1}{2}\left(\chi_{i}^{k} e_{k j}+\chi_{j}^{k} e_{i k}\right)+O\left(e^{2}\right) .
$$

Recall that indices are raised with $G^{i j}$.

We now explicitly check the background independence to quadratic order by confirming that $\delta S=0$ up to terms cubic in fields under the variations

$$
\delta e_{i j}=\chi_{i j}-\frac{1}{2}\left(\chi_{i}^{k} e_{k j}-\chi_{j}^{k} e_{i k}\right), \quad \delta E_{i j}=-\chi_{i j} .
$$

The background shift changes $G^{i j}$ by

$$
\delta G^{i j}=\chi^{(i j)} \equiv \frac{1}{2}\left(\chi^{i j}+\chi^{j i}\right)
$$

with $\chi^{i j}=G^{i k} \chi_{k l} G^{l j}$. The full variation of $e_{i j}$ is needed for quadratic terms in the action, but only the leading part is needed for the cubic terms. For the computation it is useful to recall that $\tilde{\partial}^{i}=\frac{1}{2}\left(\bar{D}^{i}-D^{i}\right)$, which implies that

$$
\delta D_{i}=\frac{1}{2} \chi_{i k}\left(\bar{D}^{k}-D^{k}\right), \quad \delta \bar{D}_{i}=\frac{1}{2} \chi_{k i}\left(D^{k}-\bar{D}^{k}\right) .
$$


These lead to

$$
\delta \square \equiv \delta\left(G^{i j} D_{i} D_{j}\right)=\chi^{i j} D_{i} \bar{D}_{j}
$$

This identity in fact suffices to prove the background independence for the terms quadratic in the dilaton in the action (2.1). Indeed, the following variations cancel

$$
\begin{aligned}
\delta(-4 d \square d) & =-4 d \chi^{i j} D_{i} \bar{D}_{j} d, \\
\delta\left(4 e_{i j} d D^{i} \bar{D}^{j} d\right) & =4 \chi_{i j} d D^{i} \bar{D}^{j} d .
\end{aligned}
$$

Checking the terms linear in $d$ is relatively straightforward. For structures quadratic in $e$, the variation of the bilinear terms gives

$$
\delta \mathcal{L}^{(2)}=\frac{1}{4} \chi^{k l} e^{i j} D_{k} \bar{D}_{l} e_{i j}+\frac{1}{4} \chi^{m j} D_{m} e_{i j} \bar{D}_{k} e^{i k}+\frac{1}{4} \chi^{i m} \bar{D}_{m} e_{i j} D_{k} e^{k j} .
$$

One can verify that, up to total derivatives, this variation is cancelled by the variation of the terms cubic in $e_{i j}$. We note that these computations do not require using the constraint $\partial_{i} \tilde{\partial}^{i}=0$.

Having checked background independence, we can introduce a field $\mathcal{E}_{i j}$ that combines the background $E_{i j}$ and the fluctuation $e_{i j}$ and is background independent in the sense that it is invariant under the transformations (2.8). We find that

$$
\mathcal{E}_{i j} \equiv E_{i j}+e_{i j}+\frac{1}{2} e_{i}{ }^{k} e_{k j}+\mathcal{O}\left(e^{3}\right),
$$

has this property. Indeed, under (2.8),

$$
\begin{aligned}
\delta \mathcal{E}_{i j} & =\delta E_{i j}+\delta e_{i j}+\frac{1}{2} \delta e_{i}{ }^{k} e_{k j}+\frac{1}{2} e_{i}{ }^{k} \delta e_{k j}+\mathcal{O}\left(e^{2}\right) \\
& =-\chi_{i j}+\chi_{i j}-\frac{1}{2}\left(\chi_{i}{ }^{k} e_{k j}-\chi^{k}{ }_{j} e_{i k}\right)+\frac{1}{2} \chi_{i}{ }^{k} e_{k j}+\frac{1}{2} e_{i}{ }^{k} \chi_{k j}+\mathcal{O}\left(e^{2}\right) \\
& =0+\mathcal{O}\left(e^{2}\right) .
\end{aligned}
$$

Note that the index contraction $e_{i}{ }^{k} e_{k j}$ on the right-hand side of (2.14) breaks the $O(D, D)$ covariance, as will be discussed in subsection 2.4. These are the first terms in the full non-linear form of $\mathcal{E}$ in terms of $E$ and $e$ that was found in [3, 15] and which will be discussed in the next subsection. It was shown in [3] that upon setting all $\tilde{\partial}$ derivatives equal to zero, the field $\mathcal{E}$ has the familiar gauge transformations associated with gravity and the antisymmetric tensor field.

\subsection{The gauge transformations in background independent form}

In [2], the action to cubic order in the fields was found to be (2.1), and the corresponding gauge transformations preserving this action were found to linear order in the fields. Reference [3] considered the situation in which the fields and gauge parameters are restricted to lie in a totally null subspace of the doubled space i.e. a subspace in which all vectors are null and mutually orthogonal. Such a restriction arises when the fields and parameters are all independent of $\tilde{x}$, or any configuration related to this by T-duality. The restriction guarantees that the strong form of the $\partial_{M} \partial^{M}=0$ constraint holds. 
With the restriction, the full gauge transformations to all orders in the fields are in fact quadratic in the fields [3] and given by:

$$
\begin{aligned}
\delta_{\lambda} e_{i j}= & D_{i} \bar{\lambda}_{j}+\bar{D}_{j} \lambda_{i} \\
& +\frac{1}{2}(\lambda \cdot D+\bar{\lambda} \cdot \bar{D}) e_{i j}+\frac{1}{2}\left(D_{i} \lambda^{k}-D^{k} \lambda_{i}\right) e_{k j}-e_{i k} \frac{1}{2}\left(\bar{D}^{k} \bar{\lambda}_{j}-\bar{D}_{j} \bar{\lambda}^{k}\right) \\
& -\frac{1}{4} e_{i k}\left(D^{l} \bar{\lambda}^{k}+\bar{D}^{k} \lambda^{l}\right) e_{l j},
\end{aligned}
$$

where $\lambda_{i}$ and $\bar{\lambda}_{i}$ are independent real parameters. In [3] these gauge transformations were shown to close according to the so-called C-bracket. To define this bracket we introduce new gauge parameters $\xi^{i}$ and $\tilde{\xi}_{i}$ defined by

$$
\lambda_{i}=-\tilde{\xi}_{i}+E_{i j} \xi^{j}, \quad \bar{\lambda}_{i}=\tilde{\xi}_{i}+E_{j i} \xi^{j}
$$

Using these parameters we can write the gauge algebra in a manifestly $O(D, D)$ covariant form. More precisely, if we assemble $\xi^{i}$ and $\tilde{\xi}_{i}$ into the fundamental $O(D, D)$ vector

$$
\xi^{M}=\left(\begin{array}{c}
\tilde{\xi}_{i} \\
\xi^{i}
\end{array}\right),
$$

the closure of the gauge transformations defines the algebra $\left[\delta_{\xi_{1}}, \delta_{\xi_{2}}\right]=-\delta_{\left[\xi_{1}, \xi_{2}\right]_{\mathrm{C}}}$, where the C-bracket $[\cdot, \cdot]_{\mathrm{C}}$ is given by

$$
\left[\xi_{1}, \xi_{2}\right]_{\mathrm{C}}^{M}=\xi_{1}^{N} \partial_{N} \xi_{2}^{M}-\frac{1}{2} \eta^{M N} \eta_{P Q} \xi_{1}^{P} \partial_{N} \xi_{2}^{Q}-(1 \leftrightarrow 2)
$$

The bracket (2.19) was the starting point for the construction of Siegel [5]. In generalized geometry this is a bracket for a 'Courant bi-algebroid', and when restricted to fields and parameters independent of tilde coordinates, it reduces to the Courant bracket; see the discussion in [3]. The background independent form of the gauge transformations we are about to derive provide just a rewriting of the symmetry transformations, and therefore they will close according to the same bracket. Thus the gauge algebra of the background independent theory can be said to be defined by the Courant bracket.

Let us now turn to the rewriting of the gauge transformations (2.16). We first write them as

$$
\delta_{\lambda} e_{i j}=\frac{1}{2}(\lambda \cdot D+\bar{\lambda} \cdot \bar{D}) e_{i j}+\hat{D}_{i} \bar{\lambda}_{j}+\hat{\bar{D}}_{j} \lambda_{i}+\frac{1}{2}\left(\hat{D}_{i} \lambda^{k}\right) e_{k j}+\frac{1}{2} e_{i k} \hat{\bar{D}}_{j} \bar{\lambda}^{k}
$$

where

$$
\hat{D}_{i} \equiv D_{i}-\frac{1}{2} e_{i k} \bar{D}^{k}, \quad \hat{\bar{D}}_{j} \equiv \bar{D}_{j}-\frac{1}{2} e_{k j} D^{k}
$$

The full metric is $g_{i j}=G_{i j}+h_{i j}$ and the antisymmetric tensor gauge field is $b_{i j}=B_{i j}+\hat{b}_{i j}$ where $G_{i j}$ and $B_{i j}$ are constant background fields. These are combined into

$$
\mathcal{E}_{i j} \equiv E_{i j}+\check{e}_{i j}
$$

where $\check{e}_{i j}=h_{i j}+\hat{b}_{i j}$. It was shown in [3] that the field $\check{e}_{i j}$ is related to $\left(e_{i j}, d\right)$ by $\check{e}_{i j}=f_{i j}(e, d)$, where

$$
f=\left(1-\frac{1}{2} e\right)^{-1} e
$$


so that

$$
\check{e}=F e,
$$

where we use matrix notation and

$$
F \equiv\left(1-\frac{1}{2} e\right)^{-1}
$$

Then the full non-linear form of (2.14) is

$$
\mathcal{E}_{i j}=E_{i j}+F_{i}^{k}(e) e_{k j} .
$$

Here and in the rest of this subsection, indices $i, j$ are raised and lowered using the background metric $G_{i j}$.

It is shown in appendix A that for any variation or derivative

$$
\delta \mathcal{E}=\delta \check{e}=F \delta e F
$$

Rewriting the gauge transformations using this naturally involves the derivatives

$$
\begin{aligned}
\mathcal{D}_{i} & \equiv F_{i j} \hat{D}_{j}, \\
\overline{\mathcal{D}}_{i} & \equiv F_{j i} \hat{\bar{D}}_{j} .
\end{aligned}
$$

Remarkably these can be written (see appendix A) in manifestly background-independent form:

$$
\begin{aligned}
& \mathcal{D}_{i}=\partial_{i}-\mathcal{E}_{i k} \tilde{\partial}^{k}, \\
& \overline{\mathcal{D}}_{i}=\partial_{i}+\mathcal{E}_{k i} \tilde{\partial}^{k} .
\end{aligned}
$$

From these derivatives one can reconstruct the ordinary partial derivatives through the formulae

$$
\partial_{i}=\frac{1}{2}\left(\mathcal{E}_{j i} \mathcal{D}^{j}+\mathcal{E}_{i j} \overline{\mathcal{D}}^{j}\right), \quad \tilde{\partial}^{i}=\frac{1}{2}\left(-\mathcal{D}^{i}+\overline{\mathcal{D}}^{i}\right) .
$$

It is useful to rewrite the gauge transformations in terms of the gauge parameters $\xi^{i}$ and $\tilde{\xi}_{i}$ defined in (2.17) [3]. Then after some work (see appendix A) the gauge transformations take the manifestly background-independent form

$$
\delta \mathcal{E}_{i j}=\mathcal{D}_{i} \tilde{\xi}_{j}-\overline{\mathcal{D}}_{j} \tilde{\xi}_{i}+\xi^{M} \partial_{M} \mathcal{E}_{i j}+\mathcal{D}_{i} \xi^{k} \mathcal{E}_{k j}+\overline{\mathcal{D}}_{j} \xi^{k} \mathcal{E}_{i k}
$$

The gauge transformations can be expanded using the derivatives $\partial_{i}, \tilde{\partial}^{k}$ as

$$
\begin{aligned}
\delta \mathcal{E}_{i j}= & \partial_{i} \tilde{\xi}_{j}-\partial_{j} \tilde{\xi}_{i} \\
& +\tilde{\xi}_{k} \tilde{\partial}^{k} \mathcal{E}_{i j}-\tilde{\partial}^{k} \tilde{\xi}_{i} \mathcal{E}_{k j}-\tilde{\partial}^{k} \tilde{\xi}_{j} \mathcal{E}_{i k} \\
& +\xi^{k} \partial_{k} \mathcal{E}_{i j}+\partial_{i} \xi^{k} \mathcal{E}_{k j}+\partial_{j} \xi^{k} \mathcal{E}_{i k} \\
& +\mathcal{E}_{i k}\left(\tilde{\partial}^{q} \xi^{k}-\tilde{\partial}^{k} \xi^{q}\right) \mathcal{E}_{q j} .
\end{aligned}
$$

Along with the standard Lie derivative $\mathcal{L}_{\xi}$ with respect to $\xi^{i}$, we introduce a dual Lie derivative with respect to the tilde parameter $\tilde{\xi}_{i}$ :

$$
\mathcal{L}_{\tilde{\xi}} \mathcal{E}_{i j} \equiv \tilde{\xi}_{k} \tilde{\partial}^{k} \mathcal{E}_{i j}-\tilde{\partial}^{k} \tilde{\xi}_{i} \mathcal{E}_{k j}-\tilde{\partial}^{k} \tilde{\xi}_{j} \mathcal{E}_{i k}
$$


The gauge transformations (2.32) can then be written more compactly as

$$
\delta_{\xi} \mathcal{E}_{i j}=\partial_{i} \tilde{\xi}_{j}-\partial_{j} \tilde{\xi}_{i}+\mathcal{L}_{\xi} \mathcal{E}_{i j}+\mathcal{E}_{i k}\left(\tilde{\partial}^{q} \xi^{k}-\tilde{\partial}^{k} \xi^{q}\right) \mathcal{E}_{q j}+\mathcal{L}_{\tilde{\xi}} \mathcal{E}_{i j}
$$

For fields and parameters that are restricted to be independent of $\tilde{x}$ so that $\tilde{\partial}^{i}=0$, this reduces to

$$
\delta \mathcal{E}_{i j}=\mathcal{L}_{\xi} \mathcal{E}_{i j}+\partial_{i} \tilde{\xi}_{j}-\partial_{j} \tilde{\xi}_{i}
$$

which is the standard form of a diffeomorphism with the infinitesimal vector field $\xi^{i}$ and a twoform gauge transformation with the infinitesimal one-form $\tilde{\xi}_{i}$. The gauge variation of the inverse $\tilde{\mathcal{E}}^{i j} \equiv\left(\mathcal{E}^{-1}\right)_{i j}$ is then

$$
\begin{aligned}
\delta \tilde{\mathcal{E}}^{i j} & =-\tilde{\mathcal{E}}^{i k} \delta \mathcal{E}_{k l} \tilde{\mathcal{E}}^{l j} \\
& =\tilde{\partial}^{i} \xi^{j}-\tilde{\partial}^{j} \xi^{i}+\tilde{\xi}_{k} \tilde{\partial}^{k} \tilde{\mathcal{E}}^{i j}+\tilde{\partial}^{i} \tilde{\xi}_{k} \tilde{\mathcal{E}}^{k j}+\tilde{\partial}^{j} \tilde{\xi}_{k} \tilde{\mathcal{E}}^{i k} \\
& -\tilde{\mathcal{E}}^{i k}\left(\partial_{k} \tilde{\xi}_{l}-\partial_{l} \tilde{\xi}_{k}\right) \tilde{\mathcal{E}}^{l j}+\xi^{k} \partial_{k} \tilde{\mathcal{E}}^{i j}-\tilde{\mathcal{E}}^{i k} \partial_{k} \xi^{j}-\tilde{\mathcal{E}}^{k j} \partial_{k} \xi^{i} .
\end{aligned}
$$

We can write this result as

$$
\delta \tilde{\mathcal{E}}^{i j}=\tilde{\partial}^{i} \xi^{j}-\tilde{\partial}^{j} \xi^{i}+\mathcal{L}_{\tilde{\xi}} \tilde{\mathcal{E}}^{i j}+\tilde{\mathcal{E}}^{i k}\left(\partial_{l} \tilde{\xi}_{k}-\partial_{k} \tilde{\xi}_{l}\right) \tilde{\mathcal{E}}^{l j}+\mathcal{L}_{\xi} \tilde{\mathcal{E}}^{i j}
$$

where

$$
\mathcal{L}_{\tilde{\xi}} \tilde{\mathcal{E}}^{i j}=\tilde{\xi}_{k} \tilde{\partial}^{k} \tilde{\mathcal{E}}^{i j}+\tilde{\partial}^{i} \tilde{\xi}_{k} \tilde{\mathcal{E}}^{k j}+\tilde{\partial}^{j} \tilde{\xi}_{k} \tilde{\mathcal{E}}^{i k}
$$

By comparing with (2.34) we infer that the role of $\xi$ and $\tilde{\xi}$ and of $\mathcal{E}$ and $\tilde{\mathcal{E}}$ are precisely interchanged; we shall see later that this interchange is a T-duality. For fields and parameters that are restricted to be independent of $x$ so that $\partial_{i}=0$, this reduces to

$$
\delta \tilde{\mathcal{E}}^{i j}=\tilde{\partial}^{i} \xi^{j}-\tilde{\partial}^{j} \xi^{i}+\mathcal{L}_{\tilde{\xi}} \tilde{\mathcal{E}}^{i j},
$$

which is the standard form of a diffeomorphism of $\tilde{x}_{i}$ with the infinitesimal vector field $\tilde{\xi}_{i}$ and a two-form gauge transformation with the infinitesimal one-form $\xi^{i}$. (Note that here lower indices are contravariant and upper ones covariant, so that the signs in (2.38) are the conventional ones.)

For the dilaton the full gauge transformation found in [3] is

$$
\delta_{\lambda} d=-\frac{1}{4}(D \cdot \lambda+\bar{D} \cdot \bar{\lambda})+\frac{1}{2}(\lambda \cdot D+\bar{\lambda} \cdot \bar{D}) d,
$$

and can be rewritten as

$$
\delta_{\lambda} d=-\frac{1}{2} \partial_{M} \xi^{M}+\xi^{M} \partial_{M} d .
$$

The gauge transformations are reducible - there are "symmetries of symmetries". One can verify that parameters of the form

$$
\xi^{M}=\partial^{M} \chi \quad \leftrightarrow \quad \tilde{\xi}_{i}=\partial_{i} \chi, \quad \xi^{i}=\tilde{\partial}^{i} \chi,
$$

generate gauge transformations (2.31), (2.41) that leave the fields unchanged, so that they are trivial gauge transformations. The transport term is $\xi^{M} \partial_{M} \mathcal{E}_{i j}=\partial^{M} \chi \partial_{M} \mathcal{E}_{i j}=0$ because of the strong form of the constraint. For the rest of the terms a small calculation is needed:

$$
\begin{aligned}
\delta \mathcal{E}_{i j}= & \mathcal{D}_{i} \partial_{j} \chi-\overline{\mathcal{D}}_{j} \partial_{i} \chi+\mathcal{D}_{i} \tilde{\partial}^{k} \chi \mathcal{E}_{k j}+\overline{\mathcal{D}}_{j} \tilde{\partial}^{k} \chi \mathcal{E}_{i k} \\
= & -\mathcal{E}_{i k} \tilde{\partial}^{k} \partial_{j} \chi-\mathcal{E}_{k j} \tilde{\partial}^{k} \partial_{i} \chi+\partial_{i} \tilde{\partial}^{k} \chi \mathcal{E}_{k j}+\partial_{j} \tilde{\partial}^{k} \chi \mathcal{E}_{i k} \\
& -\mathcal{E}_{i q}\left(\tilde{\partial}^{q} \tilde{\partial}^{k} \chi\right) \mathcal{E}_{k j}+\mathcal{E}_{q j}\left(\tilde{\partial}^{q} \tilde{\partial}^{k} \chi\right) \mathcal{E}_{i k}=0 .
\end{aligned}
$$




\subsection{Constructing the action}

The analysis in the previous subsection led to a background independent field $\mathcal{E}_{i j}$ defined in (2.26) (see also (2.14)). In this section, we use this to construct a background independent action that agrees with (2.1) to cubic order. There is, however, a significant constraint. The action (2.1) only has $O(D, D)$ consistent index contractions while the redefinition (2.26) contains $O(D, D)$ violating contractions. It is a stringent consistency test that the action in terms of $\mathcal{E}_{i j}$ should only have $O(D, D)$ consistent contractions. We find a non-polynomical action that is background independent and has only consistent index contractions. In the following sections, we check that this action is duality covariant and gauge invariant, so that it must be the full non-linear action.

Given that the action to be constructed involves $\mathcal{E}$ it is natural to modify the derivatives (2.2) and use the calligraphic derivatives (2.29) introduced in the previous subsection. From these derivatives one can reconstruct the ordinary partial derivatives through the formulae (2.30). The constraint $\partial_{M} A \partial^{M} B=0$, or equivalently $\partial_{i} A \tilde{\partial}^{i} B+\tilde{\partial}^{i} A \partial_{i} B=0$ takes a simple form using calligraphic derivatives. A short calculation shows that it is equivalent to

$$
\mathcal{D}^{i} A \mathcal{D}_{i} B=\overline{\mathcal{D}}^{i} A \overline{\mathcal{D}}_{i} B
$$

We also must have for any $A$ :

$$
\partial_{i} \tilde{\partial}^{i} A=0
$$

or equivalently $\partial_{M} \partial^{M} A=0$. Using (2.30) this constraint can be written with calligraphic derivatives as

$$
\mathcal{E}_{i j}\left(\overline{\mathcal{D}}^{j} \overline{\mathcal{D}}^{i}-\mathcal{D}^{i} \mathcal{D}^{j}-\overline{\mathcal{D}}^{j} \mathcal{D}^{i}+\mathcal{D}^{i} \overline{\mathcal{D}}^{j}\right) A=0
$$

We define $g^{i j}$ as the inverse of the metric $g_{i j}=\frac{1}{2}\left(\mathcal{E}_{i j}+\mathcal{E}_{j i}\right)$. A short calculation shows that

$$
g^{i j}=G^{i j}-e^{(i j)}+\frac{1}{4} e^{i k} e_{k}^{j}+\frac{1}{4} e^{k i} e_{k}{ }^{j}+\mathcal{O}\left(e^{3}\right) .
$$

Indices on the fluctuation field $e_{i j}$ are raised with the constant background (inverse) metric $G^{i j}$. The calligraphic derivatives can be written in terms of $D, \bar{D}$ derivatives and fluctuations

$$
\begin{aligned}
& \mathcal{D}_{i}=D_{i}-\frac{1}{2} e_{i k}\left(\bar{D}^{k}-D^{k}\right)-\frac{1}{4} e_{i}^{k} e_{k l}\left(\bar{D}^{l}-D^{l}\right)+\mathcal{O}\left(e^{3}\right), \\
& \overline{\mathcal{D}}_{i}=\bar{D}_{i}+\frac{1}{2} e_{k i}\left(\bar{D}^{k}-D^{k}\right)+\frac{1}{4} e_{k}{ }^{l} e_{l i}\left(\bar{D}^{k}-D^{k}\right)+\mathcal{O}\left(e^{3}\right) .
\end{aligned}
$$

The indices on the calligraphic derivatives will be raised with the full metric $g^{i j}$. We thus find:

$$
\begin{aligned}
& \mathcal{D}^{i} \equiv g^{i j} \mathcal{D}_{j}=D^{i}-\frac{1}{2} e^{i j} \bar{D}_{j}-\frac{1}{2} e^{j i} D_{j}+\mathcal{O}\left(e^{2}\right), \\
& \overline{\mathcal{D}}^{i} \equiv g^{i j} \overline{\mathcal{D}}_{j}=\bar{D}^{i}-\frac{1}{2} e^{k i} D_{k}-\frac{1}{2} e^{i k} \bar{D}_{k}+\mathcal{O}\left(e^{2}\right) .
\end{aligned}
$$

An action written only using the calligraphic derivatives, $g^{i j}, \mathcal{E}_{i j}$ and $d$ will be manifestly background independent. As discussed in the following subsection, it will be duality covariant provided the index contractions all satisfy the rules of contraction formulated in [2]. Our strategy now is to 
seek such a background independent duality covariant action that agrees with the action (2.1) to cubic order. The dilaton theorem states that a constant shift of the dilaton is equivalent to a change of the coupling constant. This theorem is manifest in actions where the dilaton appears in an exponential prefactor that multiplies all terms in the Lagrangian, and all other occurrances of the dilaton involve its derivatives. We thus aim for an action where the overall multiplicative factor takes the conventional form $e^{-2 d}$ and elsewhere the dilaton appears with derivatives.

We now begin the computation. The $(-4 d \square d)$ term in the action (2.1) could come from a term $4 e^{-2 d} g^{i j} \mathcal{D}_{i} d \mathcal{D}_{j} d$. Expansion in fluctuations gives the desired quadratic term and extra cubic terms:

$$
4 e^{-2 d} g^{i j} \mathcal{D}_{i} d \mathcal{D}_{j} d=-4 d \square d+4 e^{i j} d D_{i} \bar{D}_{j} d+4 d^{2} \square d-2 d^{2} D^{i} \bar{D}^{j} e_{i j}+(\mathrm{td}),
$$

where $(\mathrm{td})$ stands for $\partial_{i}$ and $\tilde{\partial}^{i}$ total derivatives, $(\mathrm{td})=\partial_{M} v^{M}$ for some $v^{M}$. These terms can be ignored as we integrate to form the action. For the quadratic terms mixing the dilaton and $e_{i j}$ we use

$$
\begin{aligned}
e^{-2 d}\left(\mathcal{D}^{i} d \overline{\mathcal{D}}^{j} \mathcal{E}_{i j}+\overline{\mathcal{D}}^{j} d \mathcal{D}^{i} \mathcal{E}_{i j}\right)= & -2 d D^{i} \bar{D}^{j} e_{i j}+d e^{i j}\left(D_{i} D^{k} e_{k j}+\bar{D}_{j} \bar{D}^{k} e_{i k}\right) \\
& +\frac{1}{2} d\left(D^{i} e_{i j}\right)^{2}+\frac{1}{2} d\left(\bar{D}^{j} e_{i j}\right)^{2} \\
& +\frac{1}{2} d \bar{D}^{j} e^{i l} \bar{D}_{l} e_{i j}+\frac{1}{2} d D^{i} e^{k j} D_{k} e_{i j}+2 d^{2} D^{i} \bar{D}^{j} e_{i j}+(\mathrm{td}) .
\end{aligned}
$$

To obtain the terms quadratic in $e_{i j}$ we need three structures

$$
\begin{aligned}
\frac{1}{4} e^{-2 d} g^{k l} \mathcal{D}^{j} \mathcal{E}_{i k} \mathcal{D}^{i} \mathcal{E}_{j l}= & \frac{1}{4}\left(D^{i} e_{i k}\right)^{2}-\frac{1}{4} e_{i j} D^{k} e^{i l} \bar{D}^{j} e_{k l}-\frac{1}{2} d D^{j} e_{i k} D^{i} e_{j}{ }^{k}+(\mathrm{td}), \\
\frac{1}{4} e^{-2 d} g^{k l} \overline{\mathcal{D}}^{j} \mathcal{E}_{k i} \overline{\mathcal{D}}^{i} \mathcal{E}_{l j}= & \frac{1}{4}\left(\bar{D}^{i} e_{k i}\right)^{2}-\frac{1}{4} e_{i j} D^{i} e_{k l} \bar{D}^{l} e^{k j}-\frac{1}{2} d \bar{D}^{j} e^{k i} \bar{D}_{i} e_{k j}+(\mathrm{td}), \\
-\frac{1}{4} e^{-2 d} g^{i k} g^{j l} \mathcal{D}^{p} \mathcal{E}_{k l} \mathcal{D}_{p} \mathcal{E}_{i j}= & \frac{1}{4} e^{i j} \square e_{i j}+\frac{1}{4} e^{i j} D_{i} e^{k l} \bar{D}_{j} e_{k l} \\
& +\frac{1}{4} d D^{i} e^{j k} D_{i} e_{j k}+\frac{1}{4} d \bar{D}^{i} e^{j k} \bar{D}_{i} e_{j k}+(\mathrm{td}) .
\end{aligned}
$$

In all of the above equations we are ignoring terms of higher order than cubic in the fields. We also note that here the constraint $\partial_{M} \partial^{M}=0$ is not used. The five structures above, added together, give the action

$$
\begin{aligned}
S=\int d x d \tilde{x} e^{-2 d}[ & -\frac{1}{4} g^{i k} g^{j l} \mathcal{D}^{p} \mathcal{E}_{k l} \mathcal{D}_{p} \mathcal{E}_{i j}+\frac{1}{4} g^{k l}\left(\mathcal{D}^{j} \mathcal{E}_{i k} \mathcal{D}^{i} \mathcal{E}_{j l}+\overline{\mathcal{D}}^{j} \mathcal{E}_{k i} \overline{\mathcal{D}}^{i} \mathcal{E}_{l j}\right) \\
& \left.+\left(\mathcal{D}^{i} d \overline{\mathcal{D}}^{j} \mathcal{E}_{i j}+\overline{\mathcal{D}}^{i} d \mathcal{D}^{j} \mathcal{E}_{j i}\right)+4 \mathcal{D}^{i} d \mathcal{D}_{i} d\right]
\end{aligned}
$$

and, by construction, reproduce all quadratic terms in the action (2.1). Remarkably, they also reproduce precisely the cubic terms in (2.1), with no further terms needed!

Terms have been grouped in parentheses to make the $\mathbb{Z}_{2}$ symmetry of the action manifest. This symmetry, discussed in [2], exchanges the indices in $\mathcal{E}$, exchanges barred and unbarred derivatives, and leaves the dilaton invariant. For the first and last term the constraint (2.43) is needed to guarantee the $\mathbb{Z}_{2}$ symmetry of the action. 
Making all metrics explicit, the action is

$$
\begin{aligned}
S=\int d x d \tilde{x} e^{-2 d}[ & -\frac{1}{4} g^{i k} g^{j l} g^{p q}\left(\mathcal{D}_{p} \mathcal{E}_{k l} \mathcal{D}_{q} \mathcal{E}_{i j}-\mathcal{D}_{i} \mathcal{E}_{l p} \mathcal{D}_{j} \mathcal{E}_{k q}-\overline{\mathcal{D}}_{i} \mathcal{E}_{p l} \overline{\mathcal{D}}_{j} \mathcal{E}_{q k}\right) \\
& \left.+g^{i k} g^{j l}\left(\mathcal{D}_{i} d \overline{\mathcal{D}}_{j} \mathcal{E}_{k l}+\overline{\mathcal{D}}_{i} d \mathcal{D}_{j} \mathcal{E}_{l k}\right)+4 g^{i j} \mathcal{D}_{i} d \mathcal{D}_{i} d\right]
\end{aligned}
$$

This action is background-independent and, as we will show in the next subsection, it is invariant under T-duality. Any non-trivial background independent term with two derivatives that could be added to $S$ would also contribute to the quadratic and cubic actions and so would spoil the agreement with (2.1). Thus we conclude that $S$ is the complete background independent action! Indeed, we will establish that this is the case, and in particular show that $S$ is invariant under the gauge transformations (2.31), is duality invariant, and reduces to the standard action for suitably restricted fields.

\section{$2.4 \mathrm{O}(\mathrm{D}, \mathrm{D})$ invariance}

The action (2.1) was proven in [2] to possess T-duality covariance. In that work a notation was used that allowed to deal with T-duality transformations in a theory with both compact and non-compact directions. The spacetime has dimension $D=n+d$ and is the product of $n$-dimensional Minkowski space $\mathbb{R}^{n-1,1}$ and a torus $T^{d}$. Although we write $O(D, D)$ matrices, the ones that are used describe T-dualities that belong to the $O(d, d)$ subgroup associated with the torus. The subgroup preserving the periodicity conditions of the doubled torus is the discrete group $O(d, d ; \mathbb{Z})$, and this is the proper T-duality group. It is interesting to note, however, that our action could also be used for a situation in which all coordinates are non-compact but are nonetheless doubled, so that we have $2 D$ non-compact coordinates $x, \tilde{x}$. For such a set-up, the theory formally has a continuous $O(D, D)$ symmetry and for this reason we will not always distinguish here between the discrete and continuous groups, and will refer to both as T-dualities. We stress, however, that the case relevant to string theory is that in which there are $d$ compact coordinates and their doubles, with T-duality group $O(d, d ; \mathbb{Z})$.

The $O(D, D)$ matrices $h$ take the form

$$
h=\left(\begin{array}{ll}
a & b \\
c & d
\end{array}\right), \quad h^{-1}=\left(\begin{array}{ll}
d^{t} & b^{t} \\
c^{t} & a^{t}
\end{array}\right), \quad h^{t} \eta h=\eta, \quad \eta=\left(\begin{array}{ll}
0 & I \\
I & 0
\end{array}\right) .
$$

If the background field $E$ is viewed as a parameter in the action (2.1), then any change of its value would represent, a priori, a different theory. The $T$-duality covariance of (2.1) established the equivalence of two such actions written with different backgrounds $E$ and $E^{\prime}$ related by T-duality. Of course, if we transform both the background and the fluctuations, we get an invariance of the action. In a background independent formulation, like that of (2.54), the invariance is natural since we transform the full fields that include background and fluctuations. The doubled coordinates $X^{M}$ transform linearly under $O(D, D)$

$$
X^{\prime} \equiv\left(\begin{array}{l}
\tilde{x}^{\prime} \\
x^{\prime}
\end{array}\right)=h X=\left(\begin{array}{ll}
a & b \\
c & d
\end{array}\right)\left(\begin{array}{l}
\tilde{x} \\
x
\end{array}\right) .
$$

The derivatives $\partial_{M}=\left(\tilde{\partial}^{i}, \partial_{i}\right)$ then transform as

$$
\partial^{\prime}=\left(h^{-1}\right)^{t} \partial .
$$


It was argued in [2] that the dilaton should be an $O(D, D)$ 'scalar':

$$
d^{\prime}\left(X^{\prime}\right)=d(X)
$$

while the transformation of $\mathcal{E}_{i j}$ should take the fractional linear form

$$
\mathcal{E}^{\prime}\left(X^{\prime}\right)=(a \mathcal{E}(X)+b)(c \mathcal{E}(X)+d)^{-1},
$$

when written in terms of $D \times D$ matrices. For the special case in which the fields are independent of the toroidal coordinates and their duals, these are the familiar Buscher transformations.

It is useful to introduce matrices $M(X)$ and $\bar{M}(X)$ that control the transformations of certain tensors:

$$
M(X) \equiv d^{t}-\mathcal{E}(X) c^{t}, \quad \bar{M}(X) \equiv d^{t}+\mathcal{E}^{t}(X) c^{t} .
$$

Similar matrices were defined in [2] but with the constant background $E$ instead of the positiondependent $\mathcal{E}(X)$. We record some useful identities:

$$
\begin{aligned}
b^{t}-\mathcal{E} a^{t} & =-M(X) \mathcal{E}^{\prime}, \\
b^{t}+\mathcal{E}^{t} a^{t} & =\bar{M}(X) \mathcal{E}^{\prime t} .
\end{aligned}
$$

The $O(D, D)$ transformations of the inverse metric $g^{-1}$ can be given in two equivalent forms, one in terms of $M$ and one in terms of $\bar{M}$. The transformations are found following the arguments used in [1] and give

$$
g^{-1}=\left(\bar{M}^{t}\right)^{-1} g^{-1} \bar{M}^{-1}, \quad g^{-1}=\left(M^{t}\right)^{-1} g^{\prime-1} M^{-1} .
$$

Here $M$ and $\bar{M}$ depend on $X, g$ depends on $X$ and $g^{\prime}$ on $X^{\prime}$, so that e.g. the second expression is $g^{-1}(X)=\left(M^{t}\right)^{-1}(X) g^{-1}\left(X^{\prime}\right) M^{-1}(X)$. The matrices $M$ and $\bar{M}$ also control the transformation of the calligraphic derivatives:

$$
\mathcal{D}_{i}=M_{i}^{k} \mathcal{D}_{k}^{\prime}, \quad \overline{\mathcal{D}}_{i}=\bar{M}_{i}^{k} \overline{\mathcal{D}}_{k}^{\prime} .
$$

We will refer to quantities transforming in this way with the matrices $M$ and $\bar{M}$ acting on the indices as transforming covariantly or tensorially under $O(D, D)$. Our definition may be a little perverse, as it differs from the usual usage for tensor representations of $O(D, D)$ in which transformations such as (2.57) would be termed covariant. Then the inverse metric and the calligraphic derivatives transform tensorially. The transformation properties can be indicated using barred and unbarred indices, as in [2]. In this notation, we use lower unbarred indices for indices transforming with $M$, lower barred indices for indices transforming with $\bar{M}$, upper unbarred indices for indices transforming with $M^{-1}$ and upper barred indices for indices transforming with $\bar{M}^{-1}$.

In this terminology, an $O(D, D)$ tensor $T_{i_{1} \ldots i_{p}, \bar{j}_{1} \ldots \bar{j}_{q}}(X)$ has a number of unbarred and barred indices and transforms as follows:

$$
T_{i_{1} \ldots i_{p}, \bar{j}_{1} \ldots \bar{j}_{q}}(X)=M_{i_{1}}^{k_{1}} \ldots M_{i_{p}}^{k_{p}} \bar{M}_{\bar{j}_{1}}^{\bar{l}_{1}} \ldots \bar{M}_{\bar{j}_{q}}^{\bar{l}_{q}} T_{k_{1} \ldots k_{p}, \bar{l}_{1} \ldots \bar{l}_{q}}^{\prime}\left(X^{\prime}\right) .
$$

Here and throughout this section, $M=M(X)$ and $\bar{M}=\bar{M}(X)$ depend on $X$, not $X^{\prime}$. In other sections of the paper, we drop the bars and just write $T_{i_{1} \ldots i_{p}, j_{1} \ldots j_{q}}(X)$. An $O(D, D)$ tensor with upper 
indices transforms as

$$
U^{i_{1} \ldots i_{p}, \bar{j}_{1} \ldots \bar{j}_{q}}(X)=U^{\prime k_{1} \ldots k_{p}, \bar{l}_{1} \ldots \bar{l}_{q}}\left(X^{\prime}\right)\left(M^{-1}\right)_{k_{1}}^{i_{1}} \ldots\left(M^{-1}\right)_{k_{p}}^{i_{p}}\left(\bar{M}^{-1}\right)_{\bar{l}_{1}}^{\bar{j}_{1}} \ldots\left(\bar{M}^{-1}\right)_{\bar{l}_{q}}^{\bar{j}_{q}}
$$

With index notation and explicit barred and unbarred indices, the transformation of $g^{-1}$ in (2.63) is:

$$
g^{\bar{i} \bar{j}}=\left(\bar{M}^{t^{-1}}\right)_{\bar{p}}^{\bar{i}} g^{\prime} \bar{p} \bar{q}\left(\bar{M}^{-1}\right)_{\bar{q}}^{\bar{j}}, \quad g^{i j}=\left(M^{t^{-1}}\right)_{p}^{i} g^{p q}\left(M^{-1}\right)_{q}^{j}
$$

or eliminating the transposes,

$$
g^{\bar{i} \bar{j}}=\left(\bar{M}^{-1}\right)_{\bar{p}}^{\bar{i}} g^{\prime} \bar{p} \bar{q}\left(\bar{M}^{-1}\right)_{\bar{q}}^{\bar{j}}, \quad g^{i j}=\left(M^{-1}\right)_{p}^{i} g^{\prime p q}\left(M^{-1}\right)_{q}^{j} .
$$

Note that the inverse metric can be viewed as an object with two unbarred upper indices or two barred upper indices. This means that $g^{\bar{i}} \bar{j}=g^{i j}$ when $\bar{i}=i$ and $\bar{j}=j$. The transformations (2.65) and (2.66) are then consistent with using the metric $g$ to raise and lower indices. Our calligraphic derivatives transform as (2.64), so the index on the $\overline{\mathcal{D}}$ is of barred type and the index on $\mathcal{D}$ is unbarred.

The field $\mathcal{E}(X)$ transforms projectively, as indicated in (2.60), but variations of the field transform as an $O(D, D)$ tensor. Defining $\mathcal{E}^{\prime}+\delta \mathcal{E}^{\prime}$ as the image of $\mathcal{E}+\delta \mathcal{E}$ under an $O(D, D)$ transformation, one finds that $\delta \mathcal{E}_{i j}(X)$ and $\delta \mathcal{E}_{i j}^{\prime}\left(X^{\prime}\right)$ are related by

$$
\delta \mathcal{E}(X)=M(X) \delta \mathcal{E}^{\prime}\left(X^{\prime}\right) \bar{M}^{t}(X)
$$

which means that the first index in $\delta \mathcal{E}$ is unbarred and the second is barred. This relation applies to any variation or derivative:

$$
\partial_{i} \mathcal{E}=M \partial_{i} \mathcal{E}^{\prime} \bar{M}^{t}, \quad \tilde{\partial}^{i} \mathcal{E}=M \tilde{\partial}^{i} \mathcal{E}^{\prime} \bar{M}^{t},
$$

and therefore it also applies for calligraphic derivatives in the form

$$
\mathcal{D}_{i} \mathcal{E}=M \mathcal{D}_{i} \mathcal{E}^{\prime} \bar{M}^{t}, \quad \overline{\mathcal{D}}_{i} \mathcal{E}=M \overline{\mathcal{D}}_{i} \mathcal{E}^{\prime} \bar{M}^{t}
$$

Note that the $\mathcal{D}$ and $\overline{\mathcal{D}}$ derivatives are identical on both sides of these transformations; the $\mathcal{E}$ 's within the derivatives have not been transformed. Inserting all indices in the above and using the transformation (2.64) of the calligraphic derivatives

$$
\mathcal{D}_{i} \mathcal{E}_{j k}=M_{i}{ }^{q} M_{j}{ }^{p} \bar{M}_{k}^{\ell} \mathcal{D}_{q}^{\prime} \mathcal{E}_{p \ell}^{\prime}, \quad \overline{\mathcal{D}}_{i} \mathcal{E}_{j k}=\bar{M}_{i}{ }^{q} M_{j}{ }^{p} \bar{M}_{k}{ }^{\ell} \overline{\mathcal{D}}_{q}^{\prime} \mathcal{E}_{p \ell}^{\prime}
$$

Thus calligraphic derivatives of $\mathcal{E}$ are $O(D, D)$ tensors! With barred and unbarred indices, these are $\mathcal{D}_{i} \mathcal{E}_{j \bar{k}}$ and $\overline{\mathcal{D}}_{\bar{i}} \mathcal{E}_{j \bar{k}}$.

Using barred and unbarred indices, the Lagrangian in (2.54) can be written as

$$
\begin{aligned}
\mathcal{L}=e^{-2 d}[ & -\frac{1}{4} g^{i k} g^{\bar{j} \bar{l}} \mathcal{D}^{p} \mathcal{E}_{k \bar{l}} \mathcal{D}_{p} \mathcal{E}_{i \bar{j}}+\frac{1}{4}\left(g^{\bar{k} \bar{l}} \mathcal{D}^{j} \mathcal{E}_{i \bar{k}} \mathcal{D}^{i} \mathcal{E}_{j \bar{l}}+g^{k l} \overline{\mathcal{D}}^{\bar{j}} \mathcal{E}_{k \bar{i}} \overline{\mathcal{D}}^{\bar{i}} \mathcal{E}_{l \bar{j}}\right) \\
& \left.+\left(\mathcal{D}^{i} d \overline{\mathcal{D}}^{\bar{j}} \mathcal{E}_{i \bar{j}}+\overline{\mathcal{D}}^{\bar{i}} d \mathcal{D}^{j} \mathcal{E}_{j \bar{i}}\right)+4 \mathcal{D}^{i} d \mathcal{D}_{i} d\right]
\end{aligned}
$$

We see that all appearances of $\mathcal{E}$ are with single calligraphic derivatives, thus as $O(D, D)$ tensors. More than one derivative or no derivatives on $\mathcal{E}_{i j}$ would imply complications with $O(D, D)$ covariance. Since 
all contractions in the above Lagrangian are between upper and lower unbarred indices or between upper and lower barred indices, the Lagrangian is an $O(D, D)$ scalar:

$$
\mathcal{L}^{\prime}\left(X^{\prime}\right)=\mathcal{L}(X)
$$

As the measure is invariant, this establishes the $O(D, D)$ invariance of the action.

The $O(D, D)$ covariance of the gauge transformations is more nontrivial to verify. We have (2.31) that reads

$$
\delta \mathcal{E}_{i j}=\mathcal{D}_{i} \tilde{\xi}_{j}-\overline{\mathcal{D}}_{j} \tilde{\xi}_{i}+\mathcal{D}_{i} \xi^{k} \mathcal{E}_{k j}+\overline{\mathcal{D}}_{j} \xi^{k} \mathcal{E}_{i k}+\xi^{M} \partial_{M} \mathcal{E}_{i j}
$$

Here the gauge parameters can be grouped into the $O(D, D)$ 'vector'

$$
\xi^{M}=\left(\begin{array}{c}
\tilde{\xi}_{i} \\
\xi^{i}
\end{array}\right)
$$

We have the transformation $\xi^{\prime}=h \xi$, which implies that $\xi=h^{-1} \xi^{\prime}$. Using (2.56), we find for the components in (2.76)

$$
\tilde{\xi}=d^{t} \tilde{\xi}^{\prime}+b^{t} \xi^{\prime}, \quad \xi=c^{t} \tilde{\xi}^{\prime}+a^{t} \xi^{\prime}
$$

Using this, (2.64), (2.69), and noting that the operator $\xi^{M} \partial_{M}$ is $O(D, D)$ invariant, (2.75) becomes

$$
\begin{aligned}
M_{i}{ }^{k} \bar{M}_{j}{ }^{\ell} \delta \mathcal{E}_{k \ell}^{\prime}= & M_{i}{ }^{k} \mathcal{D}_{k}^{\prime}\left(d^{t} \tilde{\xi}^{\prime}+b^{t} \xi^{\prime}\right)_{j}-\bar{M}_{j}{ }^{\ell} \overline{\mathcal{D}}_{\ell}^{\prime}\left(d^{t} \tilde{\xi}^{\prime}+b^{t} \xi^{\prime}\right)_{i} \\
+ & M_{i}{ }^{k} \mathcal{D}_{k}^{\prime}\left(c^{t} \tilde{\xi}^{\prime}+a^{t} \xi^{\prime}\right)^{p} \mathcal{E}_{p j}+\bar{M}_{j}{ }^{\ell} \overline{\mathcal{D}}_{\ell}^{\prime}\left(c^{t} \tilde{\xi}^{\prime}+a^{t} \xi^{\prime}\right)^{k} \mathcal{E}_{i k} \\
+ & M_{i}{ }^{k} \bar{M}_{j}{ }^{\ell}\left(\xi^{\prime}{ }^{M} \partial_{M}^{\prime} \mathcal{E}_{k \ell}^{\prime}\right)
\end{aligned}
$$

Expanding out the derivatives and combining terms we readily find

$$
\begin{aligned}
M_{i}{ }^{k} \bar{M}_{j}{ }^{\ell} \delta \mathcal{E}_{k \ell}^{\prime}= & M_{i}{ }^{k} \mathcal{D}_{k}^{\prime} \tilde{\xi}_{\ell}^{\prime}(d+c \mathcal{E})_{\ell j}-\bar{M}_{j}{ }^{\ell} \overline{\mathcal{D}}_{\ell}^{\prime} \tilde{\xi}_{k}^{\prime}\left(d^{t}-\mathcal{E} c^{t}\right)_{i k} \\
& +M_{i}{ }^{k} \mathcal{D}_{k}^{\prime} \xi^{\prime \ell}(a \mathcal{E}+b)_{\ell j}+\bar{M}_{j}{ }^{\ell} \overline{\mathcal{D}}_{\ell}^{\prime} \xi^{\prime k}\left(\mathcal{E} a^{t}-b\right)_{i k} \\
& +M_{i}{ }^{k} \bar{M}_{j}{ }^{\ell}\left(\xi^{\prime M} \partial_{M}^{\prime} \mathcal{E}_{k \ell}^{\prime}\right) .
\end{aligned}
$$

We now identify using (2.61) and (2.62)

$$
\begin{aligned}
M_{i}{ }^{k} \bar{M}_{j}^{\ell} \delta \mathcal{E}_{k \ell}^{\prime}= & M_{i}{ }^{k} \bar{M}_{j}{ }^{\ell}\left(\mathcal{D}_{k}^{\prime} \tilde{\xi}_{\ell}^{\prime}-\overline{\mathcal{D}}_{\ell}^{\prime} \tilde{\xi}_{k}^{\prime}\right) \\
+ & M_{i}{ }^{k} \mathcal{D}_{k}^{\prime} \xi^{\prime p}\left(\mathcal{E}^{\prime} \bar{M}^{t}\right)_{p j}+\bar{M}_{j}^{\ell}\left(M \mathcal{E}^{\prime}\right)_{i p} \overline{\mathcal{D}}_{\ell}^{\prime} \xi^{\prime p} \\
+ & M_{i}{ }^{k} \bar{M}_{j}{ }^{\ell}\left(\xi^{\prime M} \partial_{M}^{\prime} \mathcal{E}_{k \ell}^{\prime}\right)
\end{aligned}
$$

This finally yields

$$
M_{i}{ }^{k} \bar{M}_{j}^{\ell} \delta \mathcal{E}_{k \ell}^{\prime}=M_{i}{ }^{k} \bar{M}_{j}^{\ell}\left(\mathcal{D}_{k}^{\prime} \tilde{\xi}_{\ell}^{\prime}-\overline{\mathcal{D}}_{\ell}^{\prime} \tilde{\xi}_{k}^{\prime}+\mathcal{D}_{k}^{\prime} \xi^{\prime p} \mathcal{E}_{p \ell}^{\prime}+\overline{\mathcal{D}}_{\ell}^{\prime} \xi^{\prime} p \mathcal{E}_{k p}^{\prime}+\xi^{\prime M} \partial_{M}^{\prime} \mathcal{E}_{k \ell}^{\prime}\right)
$$

Deleting the matrices $M$ and $\bar{M}$ we find that the gauge transformations for the primed variables take exactly the same form as those for the unprimed variables. This confirms the $O(D, D)$ covariance of the gauge transformations. Thus the action and the gauge transformations are consistent with $O(D, D)$ symmetry. 


\subsection{The strong constraint and restricted fields}

The strong constraint requires that all fields and gauge parameters and all of their possible products and powers are annihilated by $\partial^{M} \partial_{M}$. We will show here that this constraint implies that all fields and gauge parameters are restricted in the sense of [3]: they depend only on the coordinates of a totally null subspace $N$, so that the theory is related by an $O(D, D)$ transformation to one in which all fields and gauge parameters depend on $x$ but do not depend on $\tilde{x}$. Note that here we only consider classical field theory, so that all products of fields are conventional classical products.

The constraint means that for any two fields $A$ and $B, \partial^{M} \partial_{M} A=\partial^{M} \partial_{M} B=0$ as well as $\partial^{M} \partial_{M}(A B)=0$, which requires

$$
\partial^{M} A \partial_{M} B=0
$$

or equivalently $\partial_{i} A \tilde{\partial}^{i} B+\partial_{i} B \tilde{\partial}^{i} A=0$. Indeed, if this is true, $\partial^{M} \partial_{M}$ will annihilate all multiple products or powers of fields.

Consider first a field comprised of a single Fourier mode

$$
A\left(\tilde{x}_{i}, x^{i}\right)=A e^{i\left(\tilde{p}^{i} \tilde{x}_{i}+p_{i} x^{i}\right)} .
$$

As $(\tilde{x}, x)$ transforms as a vector under $O(D, D)$, the 'dual vector'

$$
P_{M} \equiv\left(\begin{array}{l}
\tilde{p}^{i} \\
p_{i}
\end{array}\right)
$$

also transforms as a vector under $O(D, D)$. In terms of (2.84), the constraint $\partial^{M} \partial_{M} A=0$ implies

$$
\tilde{\partial}^{i} \partial_{i} A=0 \quad \Leftrightarrow \quad \tilde{p}^{i} p_{i}=0 \quad \Leftrightarrow \quad P \cdot P \equiv \eta^{M N} P_{M} P_{N}=0 .
$$

The momentum vector $P$ corresponding to each Fourier component of each field is then a null vector. Moreover, the constraint (2.82) implies that any two momentum vectors $P_{\alpha}, P_{\beta}$ associated with two Fourier components (of the same or different fields) must be orthogonal, so that

$$
P_{\alpha} \cdot P_{\beta}=0 \quad \forall \alpha, \beta
$$

Then all momenta $P_{\alpha}$ must lie in a subspace of $\mathbb{R}^{2 D}$ that is totally null or isotropic, i.e. any two vectors in the space are both null and are mutually orthogonal.

The maximal dimension for such an isotropic subspace is $D$ and a maximal isotropic subspace is one of dimension $D$ - it is maximal as there can be no isotropic space of dimension larger than $D$. The canonical example of a maximal isotropic subspace is the subspace $T^{*} \subset \mathbb{R}^{2 D}$ spanned by $P$ 's with $\tilde{p}=0$, so that

$$
P_{M} \equiv\left(\begin{array}{c}
0 \\
p_{i}
\end{array}\right)
$$


This is the momentum space when all fields depend on $x$ and not on $\tilde{x}$, so that all momenta lie in $T^{*}$. Any isotropic subspace is a subspace of a maximal one 1 Then the strong constraint implies all momenta must lie in some maximal isotropic subspace $N$ of dimension $D$. This implies that all the fields and gauge parameters are restricted to depend only on the coordinates of a totally null $D$-dimensional subspace of the $2 D$-dimensional double space. In [3] we considered the subsector of double field theory in which all fields and parameters are restricted in this way. Thus we find that the strong constraint used here is equivalent to the restriction of all fields and parameters to a maximally isotropic subspace, as stated in [3].

Now, any maximal isotropic subspace of $\mathbb{R}^{2 D}$ is related to any other by an $O(D, D)$ transformation 2 Then the strong constraint implies that all momenta lie in a totally null subspace that is related by an $O(D, D)$ transformation to the canonical one in which $\tilde{p}=0$. As a result, any subsector of the double field theory satisfying the strong constraint is related by an $O(D, D)$ transformation to the canonical subsector of fields with no dependence on the winding coordinates.

Suppose that there are some compactified dimensions, so that $x^{i}=\left(x^{\mu}, x^{a}\right)$ where $x^{\mu}$ are $n$ coordinates of $\mathbb{R}^{n-1,1}$ and $x^{a}$ are periodic coordinates of $T^{d}$. The doubled space then has further coordinates $\tilde{x}_{i}=\left(\tilde{x}_{\mu}, \tilde{x}_{a}\right)$ with $\tilde{x}_{a}$ periodic and $\tilde{x}_{\mu}$ non-compact. As discussed in [3], the physically interesting case is that in which the fields are independent of the $\tilde{x}_{\mu}$, so that $\tilde{p}^{\mu}=0$. The toroidal momenta $p_{a}, \tilde{p}^{a}$ are discrete and lie in a discrete lattice $\Gamma \subset \mathbb{R}^{2 d}$, so that the space of allowed momenta is $\mathbb{R}^{n} \times \Gamma$. The subgroup of $O(d, d)$ preserving $\Gamma$ is the T-duality group $O(d, d ; \mathbb{Z})$. We have seen above that the strong constraint implies that the momenta lie in a totally null subspace $N$ of $\mathbb{R}^{2 D}$ that is related to the canonical one $T^{*}$ by an $O(D, D)$ transformation. This $O(D, D)$ transformation is fixed once one chooses a basis for $N$ and a basis for $T^{*}$ and demands that the $m$ 'th basis vector of $N$ is mapped to the $m^{\prime}$ th basis vector of $T^{*}$. Here, the momenta actually lie in the space $N^{\prime}=N \cap\left(\mathbb{R}^{n} \times \Gamma\right)$, while the momenta for fields independent of $\tilde{x}$ are in $T^{\prime}=T^{*} \cap\left(\mathbb{R}^{n} \times \Gamma\right)$. Choosing the basis vectors for $N$ and $T^{*}$ to be in $N^{\prime}$ and $T^{\prime}$ respectively fixes an $O(D, D)$ transformation from $N$ to $T^{*}$ that in fact takes $N^{\prime}$ to $T^{\prime}$ and so must be in the $O(d, d ; \mathbb{Z})$ subgroup of $O(D, D)$.

In summary, the strong constraint (2.82) implies we can always use the symmetry $O(D, D)$ or $O(d, d ; \mathbb{Z})$ to rotate to fields with no dependence on the winding coordinates, which is what we wanted to show.

\footnotetext{
${ }^{1}$ Suppose $E$ is an isotropic subspace of dimension $n<D$. Then it is straightforward to construct a maximal isotropic subspace $L$ containing $E$. Let $E_{\perp}$ be the space of vectors orthogonal to $E$; this contains $E$ so can be written as $E_{\perp}=E \oplus F$ for some space $F$ which has dimension $2(D-n)$. Then it is straightforward to show that $K=T^{*} \cap F$ is an isotropic subspace of dimension $D-n$ and that $L=E \oplus K$ is isotropic and of dimension $D$ and so is a maximal isotropic subspace containing $E$. Other such maximal isotropic subspaces can be obtained by acting on $L$ with $O(D, D)$ transformations preserving $E$.

${ }^{2}$ As discussed in e.g. 9], maximal isotropic subspaces can be associated with pure spinors of $S O(D, D)$. They split into two classes, corresponding to the two chiralities of $S O(D, D)$ spinors, and any maximal isotropic subspace within a given class is related to any other in that class by an $S O(D, D)$ transformation. Transformations in $O(D, D)$ relate the two classes, so that any maximal isotropic subspace can be obtained from any other by an $O(D, D)$ transformation. This result can also be seen as a corollary of Witt's theorem [28, 29.
} 


\section{Reduction to Einstein gravity and derivative expansion}

In this section we are going to check that the action (2.54) or (2.55) correctly reduces to the standard one for Einstein gravity coupled to a two-form and a dilaton when the dependence on the $\tilde{x}_{i}$ coordinates is dropped. Next, we discuss a derivative expansion in $\tilde{\partial}^{i}$ and use it to verify the full gauge invariance.

\subsection{Reduction to the Einstein-Kalb-Ramond-dilaton action}

In order to recover the standard action $S_{*}$ in (1.6) we take the action (2.55) and assume that no field depends on the $\tilde{x}$ coordinates. Thus, effectively, we set $\tilde{\partial}^{i}=0$, which implies $\mathcal{D}_{i}=\overline{\mathcal{D}}_{i}=\partial_{i}$ and $\mathcal{D}^{i}=\overline{\mathcal{D}}^{i}=\partial^{i}=g^{i j} \partial_{j}$. The resulting action $\bar{S}$ takes the form

$$
\begin{aligned}
\bar{S}=\int d x e^{-2 d}[ & -\frac{1}{4} g^{i k} g^{j l} g^{p q}\left(\partial_{p} \mathcal{E}_{k l} \partial_{q} \mathcal{E}_{i j}-\partial_{i} \mathcal{E}_{l p} \partial_{j} \mathcal{E}_{k q}-\partial_{i} \mathcal{E}_{p l} \partial_{j} \mathcal{E}_{q k}\right) \\
& \left.+2 \partial^{i} d \partial^{j} g_{i j}+4 \partial^{i} d \partial_{i} d\right]
\end{aligned}
$$

where we drop a constant volume factor $\int d \tilde{x}$. We now rewrite in terms of $g$ and $b$ :

$$
\mathcal{E}_{i j}=g_{i j}+b_{i j}
$$

No terms couple derivatives of $g$ and derivatives of $b$. We find

$$
\begin{aligned}
\bar{S}=\int d x e^{-2 d}[ & -\frac{1}{4} g^{i k} g^{j l} g^{p q}\left(\partial_{p} g_{k l} \partial_{q} g_{i j}-2 \partial_{i} g_{l p} \partial_{j} g_{k q}+\partial_{p} b_{k l} \partial_{q} b_{i j}-2 \partial_{i} b_{l p} \partial_{j} b_{k q}\right) \\
& \left.+2 \partial^{i} d \partial^{j} g_{i j}+4 \partial^{i} d \partial_{i} d\right] .
\end{aligned}
$$

Next it will be convenient to rewrite the terms involving $b_{i j}$ such that the two-form gauge invariance becomes manifest. For this we note that the gauge-invariant three-form field strength satisfies

$$
\begin{aligned}
-\frac{1}{12} H^{2} & =-\frac{1}{12} g^{i k} g^{j l} g^{p q} H_{i j p} H_{k l q} \\
& =-\frac{1}{4} g^{i k} g^{j l} g^{p q} \partial_{p} b_{k l}\left(\partial_{i} b_{j q}+\partial_{j} b_{q i}+\partial_{q} b_{i j}\right) \\
& =-\frac{1}{4} g^{i k} g^{j l} g^{p q}\left(\partial_{p} b_{k l} \partial_{q} b_{i j}-2 \partial_{i} b_{l p} \partial_{j} b_{k q}\right)
\end{aligned}
$$

after relabeling and permuting indices. Using this we get

$$
\bar{S}=\int d x e^{-2 d}\left[-\frac{1}{4} g^{i k} g^{j l} \partial^{p} g_{i j} \partial_{p} g_{k l}+\frac{1}{2} g^{p q} \partial^{i} g_{p j} \partial^{j} g_{q i}+2 \partial^{i} d \partial^{j} g_{i j}+4(\partial d)^{2}-\frac{1}{12} H^{2}\right]
$$

We now consider the action $S_{*}$, conventionally written as

$$
S_{*}=\int d x \sqrt{-g} e^{-2 \phi}\left[R+4(\partial \phi)^{2}-\frac{1}{12} H^{2}\right] .
$$

We will show that $S_{*}$ equals $\bar{S}$, after a field redefinition and discarding the integral of a total derivative. The redefinition is

$$
\sqrt{-g} e^{-2 \phi}=e^{-2 d}
$$


and gives

$$
\partial_{i} \phi=\partial_{i} d+\frac{1}{2} \Gamma_{i}, \quad \Gamma_{i}=\Gamma_{i k}^{k}=\frac{1}{2} g^{k l} \partial_{i} g_{k l}
$$

Using this in $S_{*}$ we get

$$
S_{*}=\int d x e^{-2 d}\left[R+g^{i j} \Gamma_{i} \Gamma_{j}+4 \Gamma_{i} \partial^{i} d+4(\partial d)^{2}-\frac{1}{12} H^{2}\right] .
$$

Let us focus on the Einstein term, and integrate by parts the terms with derivatives of Christoffel symbols,

$$
\begin{aligned}
\int d x e^{-2 d} R=\int d x e^{-2 d} g^{i j}\left(\partial_{k} \Gamma_{i j}^{k}-\partial_{j} \Gamma_{i k}^{k}+\Gamma_{i j}^{k} \Gamma_{k}-\Gamma_{i k}^{l} \Gamma_{j l}^{k}\right) \\
=\int d x e^{-2 d}\left[2 \partial^{i} d\left(-\Gamma_{i}+g^{j k} \Gamma_{i j k}\right)+\partial_{j} g^{i j} \Gamma_{i}-\partial_{k} g^{i j} \Gamma_{i j}^{k}\right. \\
\left.+g^{i j}\left(\Gamma_{i j}^{k} \Gamma_{k}-\Gamma_{i k}^{l} \Gamma_{i l}^{k}\right)\right]
\end{aligned}
$$

where $\Gamma_{i j k}=g_{i l} \Gamma_{j k}^{l}=\frac{1}{2}\left(\partial_{j} g_{i k}+\partial_{k} g_{j i}-\partial_{i} g_{j k}\right)$. Further simplifying the terms with the dilaton derivatives yields

$$
\begin{aligned}
& \int d x e^{-2 d} R=\int d x e^{-2 d}\left[-4 \partial^{i} d \Gamma_{i}+2 \partial^{i} d \partial^{j} g_{i j}+\partial_{j} g^{i j} \Gamma_{i}-\partial_{k} g^{i j} \Gamma_{i j}^{k}\right. \\
& \left.+g^{i j}\left(\Gamma_{i j}^{k} \Gamma_{k}-\Gamma_{i k}^{l} \Gamma_{i l}^{k}\right)\right]
\end{aligned}
$$

Using this in $S_{*}$, we find that the terms coupling $\partial^{i} d$ to $\Gamma_{i}$ cancel and we get

$$
\begin{aligned}
S_{*}=\int d x e^{-2 d} & {\left[2 \partial^{i} d \partial^{j} g_{i j}+4(\partial d)^{2}-\frac{1}{12} H^{2}\right.} \\
& \left.+\partial_{j} g^{i j} \Gamma_{i}-\partial_{k} g^{i j} \Gamma_{i j}^{k}+g^{i j}\left(\Gamma_{i j}^{k} \Gamma_{k}-\Gamma_{i k}^{l} \Gamma_{i l}^{k}\right)+g^{i j} \Gamma_{i} \Gamma_{j}\right] .
\end{aligned}
$$

A straightforward computation shows that

$$
\partial_{j} g^{i j} \Gamma_{j}-\partial_{k} g^{i j} \Gamma_{i j}^{k}+g^{i j}\left(\Gamma_{i j}^{k} \Gamma_{k}-\Gamma_{i k}^{l} \Gamma_{j l}^{k}\right)+g^{i j} \Gamma_{i} \Gamma_{j}=-\frac{1}{4} g^{i k} g^{j l} \partial^{p} g_{i j} \partial_{p} g_{k l}+\frac{1}{2} g^{p q} \partial^{i} g_{p j} \partial^{j} g_{q i} .
$$

To check this it is best to see first that all structures in (3.13) of the form $g^{i j} \partial_{k} g_{i j}$ cancel out. Then the left-over terms combine correctly. With this

$$
S_{*}=\int d x e^{-2 d}\left[2 \partial^{i} d \partial^{j} g_{i j}+4(\partial d)^{2}-\frac{1}{12} H^{2}-\frac{1}{4} g^{i k} g^{j l} \partial^{p} g_{i j} \partial_{p} g_{k l}+\frac{1}{2} g^{p q} \partial^{i} g_{p j} \partial^{j} g_{q i}\right] .
$$

This shows that the action $S_{*}$ is in fact identical, up to total derivatives, to the reduced doubled action $\bar{S}$ in (3.5). This is what we wanted to show.

Since the gauge transformations reduce for $\tilde{\partial}^{i}=0$ to the standard gauge transformations, it follows that the reduced action is gauge invariant. As a warm-up for the proof of the full gauge-invariance it will be instructive to verify this explicitly. First, we turn to the derivative expansion in $\tilde{\partial}$, which will be useful for organizing the check of gauge invariance. 


\subsection{Tilde derivative expansion, dual structure, and gauge invariance}

Our strategy here will be to work in a derivative expansion in $\tilde{\partial}$. We write the action (2.55) as

$$
S=S^{(0)}+S^{(1)}+S^{(2)},
$$

with the superscript denoting the number of $\tilde{\partial}$ derivatives in the action. In the following we also refer to the corresponding Lagrangians defined by

$$
S^{(k)}=\int d x d \tilde{x} \mathcal{L}^{(k)}, \quad k=0,1,2 .
$$

Since $\mathcal{L}^{(0)}$ contains no $\tilde{\partial}$ derivatives, $S^{(0)}$ takes the same form as the action $\bar{S}$ in equations (3.1) and (3.5), but with fields that depend both on $x^{i}$ and $\tilde{x}_{i}$ (subject to the constraint) and integration measure $d x d \tilde{x}$. We thus have the expression, corresponding to (3.1),

$$
\begin{aligned}
\mathcal{L}^{(0)}=\mathcal{L}^{(0)}[\mathcal{E}, \partial, d]=e^{-2 d}[ & -\frac{1}{4} g^{i k} g^{j l} g^{p q}\left(\partial_{p} \mathcal{E}_{k l} \partial_{q} \mathcal{E}_{i j}-\partial_{i} \mathcal{E}_{l p} \partial_{j} \mathcal{E}_{k q}-\partial_{i} \mathcal{E}_{p l} \partial_{j} \mathcal{E}_{q k}\right) \\
& \left.+2 \partial^{i} d \partial^{j} g_{i j}+4 \partial^{i} d \partial_{i} d\right] .
\end{aligned}
$$

Performing the split $\mathcal{E}_{i j}=g_{i j}+b_{i j}$, this Lagrangian can be rewritten as

$$
\mathcal{L}^{(0)}=e^{-2 d}\left(-\frac{1}{4} g^{i k} g^{j l} \partial^{p} g_{k l} \partial_{p} g_{i j}+\frac{1}{2} g^{k l} \partial^{j} g_{i k} \partial^{i} g_{j l}+2 \partial^{i} d \partial^{j} g_{i j}+4 \partial^{i} d \partial_{i} d-\frac{1}{12} H^{2}\right),
$$

which corresponds to (3.5).

Let us next consider $\mathcal{L}^{(2)}$. It can be obtained from (2.55) by collecting the terms quadratic in $\tilde{\partial}$ from the derivatives $\mathcal{D}$ and $\overline{\mathcal{D}}$. It is given by

$$
\begin{aligned}
\mathcal{L}^{(2)}=e^{-2 d}[- & \frac{1}{4} g^{i k} g^{j l} g^{p q}\left(\mathcal{E}_{p r} \mathcal{E}_{q s} \tilde{\partial}^{r} \mathcal{E}_{k l} \tilde{\partial}^{s} \mathcal{E}_{i j}-\mathcal{E}_{i r} \mathcal{E}_{j s} \tilde{\partial}^{r} \mathcal{E}_{l p} \tilde{\partial}^{s} \mathcal{E}_{k q}-\mathcal{E}_{r i} \mathcal{E}_{s j} \tilde{\partial}^{r} \mathcal{E}_{p l} \tilde{\partial}^{s} \mathcal{E}_{q k}\right) \\
& \left.-g^{i k} g^{j l}\left(\mathcal{E}_{i p} \mathcal{E}_{q j} \tilde{\partial}^{p} d \tilde{\partial}^{q} \mathcal{E}_{k l}+\mathcal{E}_{p i} \mathcal{E}_{j q} \tilde{\partial}^{p} d \tilde{\partial}^{q} \mathcal{E}_{l k}\right)+4 g^{i j} \mathcal{E}_{i k} \mathcal{E}_{j l} \tilde{\partial}^{k} d \tilde{\partial}^{l} d\right]
\end{aligned}
$$

A crucial observation is that $\mathcal{L}^{(2)}$ is the T-dual version of $\mathcal{L}^{(0)}$ in the following sense. Writing $\mathcal{E}_{i j}=(\mathcal{E})_{i j}$ with $\mathcal{E}$ a matrix, we define the inverse

$$
\tilde{\mathcal{E}}^{i j} \equiv\left(\mathcal{E}^{-1}\right)_{i j} \quad \Rightarrow \quad \tilde{\mathcal{E}}^{i k} \mathcal{E}_{k j}=\delta^{i}{ }_{j}
$$

The transformation $\mathcal{E} \rightarrow \tilde{\mathcal{E}}=\mathcal{E}^{-1}$ is a special T-duality transformation (an inversion in all circles) with $a=0, b=1, c=1$, and $d=0$. This implies $M=-\mathcal{E}$ and $\bar{M}=\mathcal{E}^{t}$. We can then use (2.63), with $\tilde{g}_{i j}$ corresponding to $g^{\prime-1}$, to derive

$$
g^{i j}=\tilde{\mathcal{E}}^{k i} \tilde{g}_{k l} \tilde{\mathcal{E}}^{l j}=\tilde{\mathcal{E}}^{i k} \tilde{g}_{k l} \tilde{\mathcal{E}}^{j l}
$$

The inverse of these relations are given by

$$
\tilde{g}_{k l}=\mathcal{E}_{k i} g^{i j} \mathcal{E}_{l j}=\mathcal{E}_{i k} g^{i j} \mathcal{E}_{j l} \text {. }
$$


The Lagrangian $\mathcal{L}^{(2)}$, as given in (3.19), can be obtained from $\mathcal{L}^{(0)}$ given in (3.17) by taking

$$
\mathcal{E}_{i j} \rightarrow \tilde{\mathcal{E}}^{i j}, \quad g^{i j} \rightarrow \tilde{g}_{i j}, \quad \partial_{i} \rightarrow \tilde{\partial}^{i}, \quad d \rightarrow d .
$$

This is checked by verifying that

$$
\begin{aligned}
\mathcal{L}^{(2)}=\mathcal{L}^{(0)}[\tilde{\mathcal{E}}, \tilde{\partial}, d]=e^{-2 d}[ & -\frac{1}{4} \tilde{g}_{i k} \tilde{g}_{j l} \tilde{g}_{p q}\left(\tilde{\partial}^{p} \tilde{\mathcal{E}}^{k l} \tilde{\partial}^{q} \tilde{\mathcal{E}}^{i j}-\tilde{\partial}^{i} \tilde{\mathcal{E}}^{l p} \tilde{\partial}^{j} \tilde{\mathcal{E}}^{k q}-\tilde{\partial}^{i} \tilde{\mathcal{E}}^{p l} \tilde{\partial}^{j} \tilde{\mathcal{E}}^{q k}\right) \\
& \left.+2 \tilde{\partial}_{i} d \tilde{\partial}_{j} \tilde{g}^{i j}+4 \tilde{\partial}^{i} d \tilde{\partial}_{i} d\right] .
\end{aligned}
$$

Consider, for example, the last term in (3.19). By virtue of (3.22) we see that it is equal to the last term in (3.24):

$$
4 e^{-2 d} g^{i j} \mathcal{E}_{i k} \mathcal{E}_{j l} \tilde{\partial}^{k} d \tilde{\partial}^{l} d=4 e^{-2 d} \tilde{g}_{i j} \tilde{\partial}^{i} d \tilde{\partial}^{j} d .
$$

It is straightforward to check that all other terms work out similarly, demonstrating the equality of this expression for $\mathcal{L}^{(2)}$ with the one given in (3.19); this is a consequence of the T-duality invariance.

Next we give the mixed action $S^{(1)}$, which is obtained from (2.55) by collecting all terms from the quadratic expressions in $\mathcal{D}, \overline{\mathcal{D}}$ that have one ordinary derivative $\partial$ and one tilde derivative $\tilde{\partial}$. The resulting Lagrangian is given by

$$
\begin{aligned}
\mathcal{L}^{(1)}=e^{-2 d}[ & \frac{1}{2} g^{i k} g^{j l} g^{p q}\left(\mathcal{E}_{p r} \tilde{\partial}^{r} \mathcal{E}_{k l} \partial_{q} \mathcal{E}_{i j}-\mathcal{E}_{l r} \tilde{\partial}^{r} \mathcal{E}_{i p} \partial_{k} \mathcal{E}_{j q}+\mathcal{E}_{r l} \tilde{\partial}^{r} \mathcal{E}_{p i} \partial_{k} \mathcal{E}_{q j}\right) \\
& +g^{i p} g^{j q}\left(\mathcal{E}_{r q} \partial_{p} d \tilde{\partial}^{r} \mathcal{E}_{i j}-\mathcal{E}_{p r} \tilde{\partial}^{r} d \partial_{q} \mathcal{E}_{i j}+\mathcal{E}_{r p} \tilde{\partial}^{r} d \partial_{q} \mathcal{E}_{i j}-\mathcal{E}_{q r} \partial_{p} d \tilde{\partial}^{r} \mathcal{E}_{j i}\right) \\
& \left.-8 g^{i j} \mathcal{E}_{i k} \tilde{\partial}^{k} d \partial_{j} d\right] .
\end{aligned}
$$

As before we decompose $\mathcal{E}_{i j}=g_{i j}+b_{i j}$, after which the Lagrangian reads

$$
\begin{aligned}
\mathcal{L}^{(1)}=e^{-2 d}[ & \frac{1}{2} g^{i k} g^{j l} g^{p q}\left(b_{p r} \tilde{\partial}^{r} b_{k l} \partial_{q} b_{i j}-2 b_{l r} \tilde{\partial}^{r} b_{i p} \partial_{k} b_{j q}+b_{p r} \tilde{\partial}^{r} g_{k l} \partial_{q} g_{i j}-2 b_{l r} \tilde{\partial}^{r} g_{i p} \partial_{k} g_{j q}\right) \\
& +g^{i k} g^{p q}\left(-\tilde{\partial}^{j} g_{i p} \partial_{k} b_{j q}-\tilde{\partial}^{j} b_{i p} \partial_{k} g_{j q}+2 b_{r q} \partial_{k} d \tilde{\partial}^{r} g_{i p}-2 b_{k r} \tilde{\partial}^{r} d \partial_{q} g_{i p}\right) \\
& \left.+2 g^{i j}\left(\partial_{j} d \tilde{\partial}^{k} b_{i k}-\tilde{\partial}^{k} d \partial_{j} b_{k i}-4 b_{i k} \tilde{\partial}^{k} d \partial_{j} d\right)\right] .
\end{aligned}
$$

Because of T-duality $\mathcal{L}^{(1)}$ is invariant under the transformation (3.23).

In order to check the full gauge invariance, we write the gauge transformation (2.34) as

$$
\delta_{\xi}=\delta_{\xi}^{(0)}+\delta_{\xi}^{(1)}
$$

where, again, the superscript denotes the number of tilde derivatives:

$$
\begin{aligned}
\delta_{\xi}^{(0)} \mathcal{E}_{i j} & =\partial_{i} \tilde{\xi}_{j}-\partial_{j} \tilde{\xi}_{i}+\mathcal{L}_{\xi} \mathcal{E}_{i j}, \\
\delta_{\xi}^{(1)} \mathcal{E}_{i j} & =-\mathcal{E}_{i k}\left(\tilde{\partial}^{k} \xi^{l}-\tilde{\partial}^{l} \xi^{k}\right) \mathcal{E}_{l j}+\mathcal{L}_{\tilde{\xi}} \mathcal{E}_{i j},
\end{aligned}
$$

using the Lie derivative (2.34). Using (2.36) we see that $\delta^{(0)}$ and $\delta^{(1)}$ are T-dual to each other. More precisely, under the transformation (3.23) together with $\xi \leftrightarrow \tilde{\xi}$ we have, for instance,

$$
\delta^{(0)} \mathcal{E}_{i j} \rightarrow \delta^{(1)} \tilde{\mathcal{E}}^{i j}
$$


Acting on (3.15) with a gauge transformation, we infer that gauge invariance requires

$$
\begin{aligned}
& \delta^{(0)} S^{(0)}=0, \\
& \delta^{(1)} S^{(2)}=0, \\
& \delta^{(0)} S^{(1)}+\delta^{(1)} S^{(0)}=0, \\
& \delta^{(1)} S^{(1)}+\delta^{(0)} S^{(2)}=0 .
\end{aligned}
$$

The first condition is the standard gauge invariance of Einstein's theory coupled to a two-form and a dilaton. The second relation is the T-dual version of this statement, and follows from the first by virtue of (3.24). Similarly, the third and fourth relation are T-dual and thus equivalent. Therefore, the only non-trivial check is, say, the third condition, which we verify explicitly.

In general, an action $\int e^{-2 d} L$ constructed from a density $e^{-2 d}$ and a Lagrangian $L$ that transforms as a scalar under diffeomorphisms is manifestly gauge invariant. More precisely, given

$$
\delta_{\xi}\left(e^{-2 d}\right)=\partial_{i}\left(\xi^{i} e^{-2 d}\right), \quad \delta_{\xi} L=\xi^{i} \partial_{i} L
$$

we find $\delta_{\xi}\left(e^{-2 d} L\right)=\partial_{i}\left(\xi^{i} e^{-2 d} L\right)$, and the action is gauge invariant (up to a surface term). The analogous statements hold for the dual diffeomorphisms parameterized by $\tilde{\xi}_{i}$. Therefore, a large part of the variation is guaranteed to combine into total derivatives, and we only have to keep track of two types of structures during the variation. First, we have to focus on the terms in the gauge transformations (2.32) that are not of the form of a Lie derivative, as the non-linear terms in (3.30). Second, we have to compute the variations of terms that involve a partial derivative, since these do not transform purely with a Lie derivative. An explicit calculation, which we defer to appendix B, shows that all these variations cancel upon use of the strong form of the constraint $\partial_{i} \tilde{\partial}^{i}=0$. This proves the gauge invariance.

\section{Towards an $\mathrm{O}(\mathrm{D}, \mathrm{D})$ geometry}

Recall that in the definition (2.65) of an $O(D, D)$ tensor, position-dependent $M(X)$ and $\bar{M}(X)$ matrices control the transformation law. Due to this $X$-dependence, neither ordinary nor calligraphic derivatives of $O(D, D)$ tensors are $O(D, D)$ tensors. We introduce here ' $O(D, D)$ covariant derivatives' that acting on $O(D, D)$ tensors yield $O(D, D)$ tensors. These derivatives will allow us to write the gauge transformations of $\mathcal{E}_{i j}$ in a form similar to that of conventional gravity. Note that we make no claims for the covariance of our derivatives under gauge transformations.

We then turn to the construction of an $O(D, D)$ scalar $\mathcal{R}$ that is also a scalar under gauge transformations: $\delta_{\xi} \mathcal{R}=\xi^{M} \partial_{M} \mathcal{R}$. This scalar $\mathcal{R}$ is built from $\mathcal{E}_{i j}$ and the dilaton $d$ and each term contains two derivatives. Together with the density $\exp (-2 d)$ this scalar can be used to construct an action. Our investigation shows that this action is equivalent to the earlier one in (2.55); the two differ by a total derivative term.

Finally, we investigate the relation between T-duality and gauge symmetries by asking to what extent the former can be seen as a special case of the latter. We stress that the gauge transformations are not diffeomorphisms on the doubled space. However, we will borrow the language of geometry, and 
refer to the transformation $\delta_{\xi} \mathcal{R}=\xi^{M} \partial_{M} \mathcal{R}$ as that of a scalar, and refer to covariance and curvatures in a mild abuse of language.

\section{1 $\mathrm{O}(\mathrm{D}, \mathrm{D})$ covariant derivatives and gauge transformations}

We have already shown in $\$ 2.4$ that the gauge transformations are $O(D, D)$ covariant. Since these transformations involve derivatives they are a natural place to investigate how to make $O(D, D)$ covariance manifest by the use of covariant derivatives. We now introduce $\eta_{i}$ and $\bar{\eta}_{i}$ gauge parameters similar to the original $\lambda_{i}$ and $\bar{\lambda}_{i}$ in (2.17), but related to $\xi^{i}$ and $\tilde{\xi}_{i}$ using $\mathcal{E}$ instead of $E$ :

$$
\eta_{i} \equiv-\tilde{\xi}_{i}+\mathcal{E}_{i j} \xi^{j}, \quad \bar{\eta}_{i} \equiv \tilde{\xi}_{i}+\xi^{j} \mathcal{E}_{j i}
$$

As before, one can also show that

$$
\xi^{i}=\frac{1}{2}\left(\eta^{i}+\bar{\eta}^{i}\right)
$$

where the indices of $\eta$ and $\bar{\eta}$ have been raised using $g^{-1}$. Using the duality transformations (2.77) of $\tilde{\xi}$ and $\xi$, it is a few lines of calculation to show that $\eta$ and $\bar{\eta}$ are $O(D, D)$ tensors (with $M=M(X), \bar{M}=$ $\bar{M}(X)$ as usual):

$$
\eta_{i}(X)=M_{i}^{p} \eta_{p}^{\prime}\left(X^{\prime}\right), \quad \bar{\eta}_{i}(X)=\bar{M}_{i}^{p} \bar{\eta}_{p}^{\prime}\left(X^{\prime}\right)
$$

We now rewrite the gauge transformation (2.31) as

$$
\begin{aligned}
\delta \mathcal{E}_{i j} & =\mathcal{D}_{i} \tilde{\xi}_{j}-\overline{\mathcal{D}}_{j} \tilde{\xi}_{i}+\xi^{M} \partial_{M} \mathcal{E}_{i j}+\mathcal{D}_{i}\left(\xi^{k} \mathcal{E}_{k j}\right)-\xi^{k} \mathcal{D}_{i} \mathcal{E}_{k j}+\overline{\mathcal{D}}_{j}\left(\xi^{k} \mathcal{E}_{i k}\right)-\xi^{k} \overline{\mathcal{D}}_{j} \mathcal{E}_{i k} \\
& =\mathcal{D}_{i}\left(\tilde{\xi}_{j}+\xi^{k} \mathcal{E}_{k j}\right)+\overline{\mathcal{D}}_{j}\left(-\tilde{\xi}_{i}+\xi^{k} \mathcal{E}_{i k}\right)+\xi^{M} \partial_{M} \mathcal{E}_{i j}-\xi^{k} \mathcal{D}_{i} \mathcal{E}_{k j}-\xi^{k} \overline{\mathcal{D}}_{j} \mathcal{E}_{i k}
\end{aligned}
$$

The above can be rewritten in terms of $\eta$ and $\bar{\eta}$ as follows:

$$
\delta \mathcal{E}_{i j}=\mathcal{D}_{i} \bar{\eta}_{j}+\overline{\mathcal{D}}_{j} \eta_{i}+\frac{1}{2}\left(\eta^{k} \mathcal{D}_{k}+\bar{\eta}^{k} \overline{\mathcal{D}}_{k}\right) \mathcal{E}_{i j}-\frac{1}{2}\left(\eta^{k}+\bar{\eta}^{k}\right) \mathcal{D}_{i} \mathcal{E}_{k j}-\frac{1}{2}\left(\eta^{k}+\bar{\eta}^{k}\right) \overline{\mathcal{D}}_{j} \mathcal{E}_{i k}
$$

Reordering we have

$$
\begin{aligned}
\delta \mathcal{E}_{i j}= & \left.\underline{\mathcal{D}_{i} \bar{\eta}_{j}}-\frac{1}{2} \underline{\left(\mathcal{D}_{i} \mathcal{E}_{k j}\right.}+\overline{\mathcal{D}}_{j} \mathcal{E}_{i k}-\overline{\mathcal{D}}_{k} \mathcal{E}_{i j}\right) \bar{\eta}^{k} \\
& \left.+\underline{\overline{\mathcal{D}}_{j} \eta_{i}}-\frac{1}{2} \underline{\left(\overline{\mathcal{D}}_{j} \mathcal{E}_{i k}\right.}+\mathcal{D}_{i} \mathcal{E}_{k j}-\mathcal{D}_{k} \mathcal{E}_{i j}\right) \eta^{k}
\end{aligned}
$$

We have underlined the terms that do not transform as $O(D, D)$ tensors. In the top line, $\mathcal{D}_{i} \bar{\eta}_{j}$ does not transform well because the derivative acts on the $\bar{M}$ that appears for the transformation of $\bar{\eta}$. All $\mathcal{D E}$ or $\overline{\mathcal{D}} \mathcal{E}$ factors are tensors (as we proved earlier), but the contraction with $\bar{\eta}$ on the first line does not respect the index type: the $k$ index is unbarred on $\mathcal{E}$ but barred in $\bar{\eta}$. Similar remarks apply to the second line. We will show below that the non-covariant terms in the variation of the first line and in that of the second line in fact cancel, so that the transformation is covariant.

It is natural to introduce Christoffel-like symbols and covariant derivatives. We define

$$
\begin{aligned}
\Gamma_{i \bar{j}}^{\bar{k}} & \equiv \frac{1}{2} g^{k l}\left(\mathcal{D}_{i} \mathcal{E}_{l j}+\overline{\mathcal{D}}_{j} \mathcal{E}_{i l}-\overline{\mathcal{D}}_{l} \mathcal{E}_{i j}\right) \\
\Gamma_{\bar{i} j}^{k} & \equiv \frac{1}{2} g^{k l}\left(\overline{\mathcal{D}}_{i} \mathcal{E}_{j l}+\mathcal{D}_{j} \mathcal{E}_{l i}-\mathcal{D}_{l} \mathcal{E}_{j i}\right) .
\end{aligned}
$$


With these we can define $O(D, D)$ covariant derivatives:

$$
\begin{aligned}
\nabla_{i} \bar{\eta}_{j} & \equiv \mathcal{D}_{i} \bar{\eta}_{j}-\Gamma_{i \bar{j}}^{\bar{k}} \bar{\eta}_{k}, \\
\bar{\nabla}_{j} \eta_{i} & \equiv \overline{\mathcal{D}}_{j} \eta_{i}-\Gamma_{\bar{j} i}^{k} \eta_{k} .
\end{aligned}
$$

These are $O(D, D)$ tensors, thus, for example $\nabla_{i} \bar{\eta}_{j}$ transforms as an $O(D, D)$ tensor with an unbarred index $i$ and a barred index $j$. With the use of the covariant derivatives the gauge transformations (4.6) become the remarkable

$$
\delta \mathcal{E}_{i j}=\nabla_{i} \bar{\eta}_{j}+\bar{\nabla}_{j} \eta_{i}
$$

This expression makes manifest the $O(D, D)$ covariance of the gauge transformations.

Additional $O(D, D)$ covariant derivatives are needed for the remaining index structures. The following definitions are $O(D, D)$ covariant:

$$
\begin{array}{ll}
\nabla_{i} \eta_{j}=\mathcal{D}_{i} \eta_{j}-\Gamma_{i j}^{k} \eta_{k}, & \Gamma_{i j}^{k}=\frac{1}{2} g^{k l} \mathcal{D}_{i} \mathcal{E}_{j l}, \\
\bar{\nabla}_{i} \bar{\eta}_{j}=\overline{\mathcal{D}}_{i} \bar{\eta}_{j}-\Gamma_{\bar{i} \bar{j}}^{\bar{k}} \bar{\eta}_{k}, & \Gamma_{\bar{i}}^{\bar{k}}=\frac{1}{2} g^{k l} \overline{\mathcal{D}}_{i} \mathcal{E}_{l j} .
\end{array}
$$

The covariant derivatives introduced above are metric compatible:

$$
\nabla_{i} g_{j k}=0
$$

This is true both if we consider $g$ to have two barred indices or two un-barred indices. For two barred indices, for example, we have

$$
\begin{aligned}
\nabla_{i} g_{\bar{j} \bar{k}} & =\mathcal{D}_{i} g_{\bar{j} \bar{k}}-\Gamma_{i \bar{j}}^{\bar{l}} g_{\overline{l k}}-\Gamma_{i \bar{k}}^{\bar{l}} g_{\bar{j} \bar{l}} \\
& =\mathcal{D}_{i} g_{\bar{j} \bar{k}}-\frac{1}{2} g^{\bar{l} \bar{p}}\left(\mathcal{D}_{i} \mathcal{E}_{p \bar{j}}+\overline{\mathcal{D}}_{j} \mathcal{E}_{i \bar{p}}-\overline{\mathcal{D}}_{p} \mathcal{E}_{i \bar{j}}\right) g_{\overline{l k}}-\frac{1}{2} g^{\bar{l} \bar{p}}\left(\mathcal{D}_{i} \mathcal{E}_{p \bar{k}}+\overline{\mathcal{D}}_{k} \mathcal{E}_{i \bar{p}}-\overline{\mathcal{D}}_{p} \mathcal{E}_{i \bar{k}}\right) g_{\bar{j} \bar{l}} \\
& =\mathcal{D}_{i} g_{\bar{j} \bar{k}}-\frac{1}{2}\left(\mathcal{D}_{i} \mathcal{E}_{k \bar{j}}+\overline{\mathcal{D}}_{j} \mathcal{E}_{i \bar{k}}-\overline{\mathcal{D}}_{k} \mathcal{E}_{i \bar{j}}\right)-\frac{1}{2}\left(\mathcal{D}_{i} \mathcal{E}_{j \bar{k}}+\overline{\mathcal{D}}_{k} \mathcal{E}_{i \bar{j}}-\overline{\mathcal{D}}_{j} \mathcal{E}_{i \bar{k}}\right) \\
& =\mathcal{D}_{i} g_{\bar{j} \bar{k}}-\frac{1}{2} \mathcal{D}_{i}\left(\mathcal{E}_{k \bar{j}}+\mathcal{E}_{j \bar{k}}\right)=0
\end{aligned}
$$

The dilaton gauge transformations (1.3) can also be rewritten in terms of covariant derivatives and the $\eta, \bar{\eta}$ gauge parameters as follows:

$$
\delta d=-\frac{1}{4}\left(\nabla_{i}-\frac{1}{2}\left(\overline{\mathcal{D}}^{p} \mathcal{E}_{i p}+4 \mathcal{D}_{i} d\right)\right) \eta^{i}-\frac{1}{4}\left(\bar{\nabla}_{i}-\frac{1}{2}\left(\mathcal{D}^{p} \mathcal{E}_{p i}+4 \overline{\mathcal{D}}_{i} d\right)\right) \bar{\eta}^{i}
$$

In finding this we used $\nabla_{i} A^{j}=\mathcal{D}_{i} A^{j}+\Gamma_{i p}^{j} A^{p}$ and a similar equation for barred derivatives. The above transformations are not as simple as those for $\mathcal{E}$ in (4.9) and perhaps indicate that defining new connections could be useful.

Let us conclude this section with a verification of the transformation properties claimed concerning the first line of (4.6). The term with the calligraphic derivative transforms as follows

$$
\left.\mathcal{D}_{i} \bar{\eta}_{j} \rightarrow M_{i}^{k} \mathcal{D}_{k}^{\prime}\left(\bar{M}_{j}^{p} \bar{\eta}_{p}^{\prime}\right)=M_{i}^{k} \bar{M}_{j}^{p} \mathcal{D}_{k}^{\prime} \bar{\eta}_{p}^{\prime}+\underline{M_{i}{ }^{k}\left(\mathcal{D}_{k}^{\prime} \bar{M}_{j}^{p}\right.}\right) \bar{\eta}_{p}^{\prime}
$$


The underlined term is a non-covariant transformation. Let us now look at the transformation of the second underlined term in the first line of (4.6):

$$
-\frac{1}{2} \bar{\eta}^{k} \mathcal{D}_{i} \mathcal{E}_{k j} \rightarrow-\frac{1}{2} \bar{\eta}^{p}\left(\bar{M}^{-1}\right)_{p}^{k} M_{k}^{r} M_{i}^{s} \bar{M}_{j}^{l}\left(\mathcal{D}_{s}^{\prime} \mathcal{E}_{r l}^{\prime}\right)
$$

This is not covariant because the $k$ indices on $\bar{\eta}$ and $\mathcal{E}$ are of different type, thus we get the matrix product $\bar{M}^{-1} M$ which is not equal to the identity. We can, however, easily find the difference from unity:

$$
\bar{M}^{-1} M=\bar{M}^{-1}(\bar{M}+(M-\bar{M}))=\mathbf{1}+\bar{M}^{-1}(M-\bar{M})=\mathbf{1}-2 \bar{M}^{-1} g c^{t},
$$

where we used (2.61) to evaluate $M-\bar{M}$. Using (2.63) we find that $\bar{M}^{-1} g=g^{\prime} \bar{M}^{t}$ and therefore

$$
\bar{M}^{-1} M=\mathbf{1}-2 g^{\prime} \bar{M}^{t} c^{t}=\mathbf{1}+2 g^{\prime} c M,
$$

where we noted that $c M=-\bar{M}^{t} c^{t}$ (using $c d^{t}=-d c^{t}$ ). Back in (4.15) we get

$$
\begin{aligned}
-\frac{1}{2} \bar{\eta}^{k} \mathcal{D}_{i} \mathcal{E}_{k j} & \rightarrow-\frac{1}{2} \bar{\eta}^{p}\left(\mathbf{1}+2 g^{\prime} c M\right)_{p}{ }^{r} M_{i}{ }^{k} \bar{M}_{j}{ }^{l}\left(\mathcal{D}_{k}^{\prime} \mathcal{E}_{r l}^{\prime}\right) \\
& =-\frac{1}{2} \bar{\eta}^{p p} M_{i}{ }^{k} \bar{M}_{j}{ }^{l}\left(\mathcal{D}_{k}^{\prime} \mathcal{E}_{p l}^{\prime}\right)-\bar{\eta}_{p}^{\prime}(c M)^{p r} \bar{M}_{j}{ }^{l} M_{i}{ }^{k}\left(\mathcal{D}_{k}^{\prime} \mathcal{E}_{r l}^{\prime}\right) .
\end{aligned}
$$

The first term on the right-hand side is covariant, the second is not. For that one we use (2.69) in reverse to transform the $\mathcal{D}^{\prime} \mathcal{E}^{\prime}$ into a $\mathcal{D}^{\prime} \mathcal{E}$ :

$$
\begin{aligned}
-\frac{1}{2} \bar{\eta}^{k} \mathcal{D}_{i} \mathcal{E}_{k j} \rightarrow & -\frac{1}{2} \bar{\eta}^{p} M_{i}{ }^{k} \bar{M}_{j}{ }^{l}\left(\mathcal{D}_{k}^{\prime} \mathcal{E}_{p l}^{\prime}\right)-\bar{\eta}_{p}^{\prime}(c M)^{p r} M_{i}{ }^{k}\left(M^{-1}\right)_{r}{ }^{q}\left(\mathcal{D}_{k}^{\prime} \mathcal{E}_{q m}\right)\left(\bar{M}^{-1}\right)_{l}{ }^{m} \bar{M}_{j}{ }^{l} \\
= & -\frac{1}{2} \bar{\eta}^{p} M_{i}{ }^{k} \bar{M}_{j}{ }^{l}\left(\mathcal{D}_{k}^{\prime} \mathcal{E}_{p l}^{\prime}\right)-M_{i}{ }^{k} \bar{\eta}_{p}^{\prime} \mathcal{D}_{k}^{\prime}(c \mathcal{E})^{p}{ }_{j} \\
= & -\frac{1}{2} \bar{\eta}^{p} M_{i}{ }^{k} \bar{M}_{j}{ }^{l}\left(\mathcal{D}_{k}^{\prime} \mathcal{E}_{p l}^{\prime}\right)-M_{i}{ }^{k} \bar{\eta}_{p}^{\prime} \mathcal{D}_{k}^{\prime} \bar{M}_{j}{ }^{p}
\end{aligned}
$$

where we used $\bar{M}^{t}=d+c \mathcal{E}$. Finally, making use of (4.14) we see that the two non-covariant terms cancel exactly! As a result, the combination that transforms covariantly is

$$
\mathcal{D}_{i} \bar{\eta}_{j}-\frac{1}{2} \bar{\eta}^{k} \mathcal{D}_{i} \mathcal{E}_{k j} \rightarrow M_{i}{ }^{k} \bar{M}_{j}^{l}\left[\left(\mathcal{D}_{k}^{\prime} \bar{\eta}_{l}^{\prime}\right)-\frac{1}{2} \bar{\eta}^{\prime p}\left(\mathcal{D}_{k}^{\prime} \mathcal{E}_{p l}^{\prime}\right)\right] .
$$

This is what we wanted to show. Note that this is the minimum combination that transforms covariantly. The covariant derivative $\nabla_{i}$ includes two additional terms that transform covariantly.

\subsection{Curvature scalar}

In this section we will construct a curvature $\mathcal{R}$ that transforms as a scalar under gauge transformations

$$
\delta_{\xi} \mathcal{R}=\xi^{M} \partial_{M} \mathcal{R}
$$

and we will show that the background-independent action (2.54) can be written in the Einstein-Hilbert like form

$$
S^{\prime}=\int d x d \tilde{x} e^{-2 d} \mathcal{R}(\mathcal{E}, d)
$$


The action (4.22) is then clearly gauge invariant as $e^{-2 d}$ is a density and $\mathcal{R}$ is a scalar. We have seen that the dilaton is an $O(D, D)$ scalar (see (2.59) $)$ and we will show that $\mathcal{R}$ is also an $O(D, D)$ scalar. It will then follow that the Lagrangian is an $O(D, D)$ scalar (as in (2.74)) and the action (4.22) is $O(D, D)$ invariant. We will refer to $\mathcal{R}$ as a curvature, even though it does not arise here from the commutator of covariant derivatives. Indeed, $\mathcal{R}$ involves the dilaton $d$ while the covariant derivatives $\nabla$ do not. A similar scalar curvature with a geometric origin was discussed by Siegel [5], and it would be interesting to understand better the relation with his curvature.

To get some feeling for the structure of this curvature scalar, we start constructing it perturbatively, as a function $\mathcal{R}(e, d)$ built using the fluctuation fields $e_{i j}$ and $d$. Expanding in fields, we write

$$
\mathcal{R}(e, d)=\mathcal{R}^{(1)}(e, d)+\mathcal{R}^{(2)}(e, d)+\ldots,
$$

where a superscript $(n)$ denotes a term of $n$ 'th order in fields. Splitting the gauge transformations similarly, $\delta=\delta^{(0)}+\delta^{(1)}+\ldots$, the conditions that $\mathcal{R}$ is a scalar give

$$
\begin{aligned}
\delta^{(0)} \mathcal{R}^{(1)} & =0 \\
\delta^{(1)} \mathcal{R}^{(1)}+\delta^{(0)} \mathcal{R}^{(2)} & =\xi^{M} \partial_{M} \mathcal{R}^{(1)}=\frac{1}{2}\left(\lambda^{i} D_{i}+\bar{\lambda}^{i} \bar{D}_{i}\right) \mathcal{R}^{(1)},
\end{aligned}
$$

where we used (2.17) to express the gauge parameters in terms of $\lambda, \bar{\lambda}$. We require that the scalar $\mathcal{R}$ is invariant under the $\mathbb{Z}_{2}$ transformation

$$
e_{i j} \rightarrow e_{j i}, \quad D_{i} \rightarrow \bar{D}_{i}, \quad \bar{D}_{i} \rightarrow D_{i}, \quad d \rightarrow d .
$$

This is a symmetry of the action so it should be a symmetry of $\mathcal{R}$. This symmetry simplifies the constraint of gauge covariance, making it sufficient to check the transformations with unbarred gauge parameter $\lambda$. From eqn. (3.27) of [2] we read

$$
\begin{array}{rlrl}
\delta^{(0)} e_{i j} & =\bar{D}_{j} \lambda_{i}, & \delta^{(1)} e_{i j} & =\frac{1}{2}\left[\left(D_{i} \lambda^{k}\right) e_{k j}-\left(D^{k} \lambda_{i}\right) e_{k j}+\lambda_{k} D^{k} e_{i j}\right], \\
\delta^{(0)} d & =-\frac{1}{4} D \cdot \lambda, \quad \delta^{(1)} d=\frac{1}{2}(\lambda \cdot D) d .
\end{array}
$$

$\mathcal{R}^{(1)}$ is linear in the fields, has two derivatives, and must be left invariant by $\delta^{(0)}$. The unique possibility, up to normalization, was determined in [3]:

$$
\mathcal{R}^{(1)}(e, d)=4 D^{2} d+D^{i} \bar{D}^{j} e_{i j}
$$

The calculation of $\mathcal{R}^{(2)}$ uses the strong form of the constraint $\partial^{M} \partial_{M}=0$. The result is:

$$
\begin{aligned}
\mathcal{R}^{(2)}(e, d)= & -4 D_{i} d D^{i} d-4 e^{i j} D_{i} \bar{D}_{j} d-2\left(D^{i} e_{i j} \bar{D}^{j} d+\bar{D}^{j} e_{i j} D^{i} d\right) \\
& -\frac{1}{2} e^{i j}\left(D_{i} D^{k} e_{k j}+\bar{D}_{j} \bar{D}^{k} e_{i k}\right)-\frac{1}{4}\left(D_{l} e^{l i} D^{k} e_{k i}+\bar{D}_{l} e^{i l} \bar{D}^{k} e_{i k}\right)-\frac{1}{4} D^{p} e^{i j} D_{p} e_{i j} .
\end{aligned}
$$

Both $\mathcal{R}^{(1)}$ and $\mathcal{R}^{(2)}$ are $O(D, D)$ scalars because all index contractions are of the right kind. Since the aim of the construction is a scalar $\mathcal{R}$ such that (4.22) is the action, we have verified that

$$
S^{\prime}=\int d x d \tilde{x} e^{-2 d} \mathcal{R}=\int d x d \tilde{x}\left(\mathcal{R}^{(2)}-2 d \mathcal{R}^{(1)}+\cdots\right)
$$


gives an action that to quadratic order in the fluctuations reproduces (2.1).

We now extend this to all orders, using the strategy of $\$ 2.3$. We find a background-independent expression that is an $O(D, D)$ scalar that agrees with $\mathcal{R}^{(1)}+\mathcal{R}^{(2)}$ to quadratic order. We then argue that this expression is the unique one with these properties and so this must be the desired curvature scalar. For any term with two derivatives on $\mathcal{E}$ or on $d, O(D, D)$ covariance requires that the second derivative cannot be a $\mathcal{D}$ but must be an $O(D, D)$ covariant derivative $\nabla$, introduced in $\$ 4.1$, We maintain the $\mathbb{Z}_{2}$ symmetry by introducing symmetrized combinations where necessary. The natural background-independent and $O(D, D)$ covariant term that agrees with the first term on the right-hand side of (4.27) to lowest order then has the following expansion to second order in the fields

$$
2 g^{i j}\left(\nabla_{i} \mathcal{D}_{j} d+\bar{\nabla}_{i} \overline{\mathcal{D}}_{j} d\right)=2\left(D^{2} d+\bar{D}^{2} d\right)-\left(D^{i} e_{i j} \bar{D}^{j} d+\bar{D}^{j} e_{i j} D^{i} d\right)-4 e^{i j} D_{i} \bar{D}_{j} d+\mathcal{O}(\text { cubic })
$$

Similarly, for the second term on the right-hand side of (4.27) we find

$$
\begin{aligned}
\frac{1}{2} g^{i k} g^{j l}\left(\nabla_{k} \overline{\mathcal{D}}_{l} \mathcal{E}_{i j}+\bar{\nabla}_{l} \mathcal{D}_{k} \mathcal{E}_{i j}\right)= & D^{i} \bar{D}^{j} e_{i j}-\frac{1}{4}\left(D^{k} e^{l j} D_{l} e_{k j}+\bar{D}^{k} e^{j l} \bar{D}_{l} e_{j k}\right) \\
& -\frac{1}{2} e^{i j}\left(D_{i} D^{k} e_{k j}+\bar{D}_{j} \bar{D}^{k} e_{i k}\right)+\mathcal{O} \text { (cubic) } .
\end{aligned}
$$

In addition to reproducing the terms linear in the fields, we also obtain the structures quadratic in the fields that contain an undifferentiated $e_{i j}$. This was necessary for the construction to succeed: such terms cannot be added in background-independent form without giving unwanted terms linear in the fluctuations upon expansion about a constant background.

So far, we have found that

$$
\begin{aligned}
\mathcal{R}^{(1)}+\mathcal{R}^{(2)}= & 2 g^{i j}\left(\nabla_{i} \mathcal{D}_{j} d+\bar{\nabla}_{i} \overline{\mathcal{D}}_{j} d\right)+\frac{1}{2} g^{i k} g^{j l}\left(\nabla_{k} \overline{\mathcal{D}}_{l} \mathcal{E}_{i j}+\bar{\nabla}_{l} \mathcal{D}_{k} \mathcal{E}_{i j}\right) \\
& +\frac{1}{4}\left(D^{k} e^{l j} D_{l} e_{k j}+\bar{D}^{k} e^{j l} \bar{D}_{l} e_{j k}\right)-\frac{1}{4}\left(D_{l} e^{l i} D^{k} e_{k i}+\bar{D}_{l} e^{i l} \bar{D}^{k} e_{i k}\right) \\
& -\frac{1}{4} D^{p} e^{i j} D_{p} e_{i j}-\left(D^{i} e_{i j} \bar{D}^{j} d+\bar{D}^{j} e_{i j} D^{i} d\right)-4 D^{i} d \bar{D}_{i} d+\mathcal{O} \text { (cubic) } .
\end{aligned}
$$

There are unique background independent expressions that give the terms on the second and third lines, at least to cubic order. Replacing the second and third lines with these gives the following candidate for the full curvature scalar:

$$
\begin{aligned}
\mathcal{R}(\mathcal{E}, d)= & 2\left(\nabla^{i} \mathcal{D}_{i} d+\bar{\nabla}^{i} \overline{\mathcal{D}}_{i} d\right)+\frac{1}{2}\left(\nabla^{i} \overline{\mathcal{D}}^{j} \mathcal{E}_{i j}+\bar{\nabla}^{j} \mathcal{D}^{i} \mathcal{E}_{i j}\right) \\
& +\frac{1}{4} g^{i j}\left(\mathcal{D}^{k} \mathcal{E}_{l j} \mathcal{D}^{l} \mathcal{E}_{k i}+\overline{\mathcal{D}}^{k} \mathcal{E}_{j l} \overline{\mathcal{D}}^{l} \mathcal{E}_{i k}\right)-\frac{1}{4} g^{i j}\left(\mathcal{D}^{l} \mathcal{E}_{l j} \mathcal{D}^{k} \mathcal{E}_{k i}+\overline{\mathcal{D}}^{l} \mathcal{E}_{j l} \overline{\mathcal{D}}^{k} \mathcal{E}_{i k}\right) \\
& -\frac{1}{4} g^{i k} g^{j l} \mathcal{D}^{p} \mathcal{E}_{i j} \mathcal{D}_{p} \mathcal{E}_{k l}-\left(\mathcal{D}^{i} \mathcal{E}_{i j} \overline{\mathcal{D}}^{j} d+\overline{\mathcal{D}}^{j} \mathcal{E}_{i j} \mathcal{D}^{i} d\right)-4 \mathcal{D}^{i} d \mathcal{D}_{i} d
\end{aligned}
$$

This is background-independent and is an $O(D, D)$ scalar that is second-order in derivatives and which reduces to $\mathcal{R}^{(1)}+\mathcal{R}^{(2)}$ upon linearization. No additional terms can be added that have all these properties: if they are not to contribute to quadratic order, they cannot have more than two $\mathcal{E}$ 's since at least one would be undifferentiated, nor can they have more than two dilatons since an 
undifferentiated dilaton violates the dilaton theorem. Then this must be the curvature scalar $\mathcal{R}$. We now proceed to check that it has the required properties.

The Lagrangian $\mathcal{L}$ for our action (2.55) differs from $e^{-2 d} \mathcal{R}$ by a total derivative, so that the action $S^{\prime}$ in (4.22) is equivalent to (2.55). Indeed, we show in appendix $\mathrm{C}$ that

$$
e^{-2 d} \mathcal{R}=\mathcal{L}+\partial_{M} \Theta^{M}
$$

where $\Theta^{M}=\left(\tilde{\theta}_{i}, \theta^{i}\right)$ transforms in the fundamental of $O(D, D)$ so that the last term on the right-hand side is an $O(D, D)$ scalar. The expressions for $\tilde{\theta}_{i}$ and $\theta^{i}$ are given in appendix $\mathrm{C}$.

A rather surprising fact is that the equation of motion of the dilaton - obtained by a straightforward variation of the action $S$ in (2.55) - is precisely $\mathcal{R}=0$ :

$$
\delta S=\int d x d \tilde{x} e^{-2 d}(-2 \delta d) \mathcal{R} .
$$

As $S$ and $S^{\prime}$ are the same up to total derivatives, both must give the same equations of motion. Varying the dilaton in $S^{\prime}$ gives

$$
\delta S^{\prime}=\int d x d \tilde{x} e^{-2 d}[(-2 \delta d) \mathcal{R}+\delta \mathcal{R}]
$$

This must agree with the variation in (4.35) and this requires that $e^{-2 d} \delta \mathcal{R}$ be a total derivative: the variation of the dilaton in $\mathcal{R}$ does not contribute to the equation of motion. The full equation of motion of the dilaton arises from the variation of $\exp (-2 d)$ alone.

Consider the case in which there is no dependence on $\tilde{x}$, so that all the terms in $\mathcal{R}$ involving $\tilde{\partial}$ drop out. A calculation similar to that in $\$ 3.1$ using $e^{-2 d}=\sqrt{-g} e^{-2 \phi}$ shows that $\mathcal{R}$ reduces to

$$
\left.\mathcal{R}\right|_{\tilde{\partial}=0}=R+4 \square \phi-4(\partial \phi)^{2}-\frac{1}{12} H^{2} .
$$

The Lagrangian $\left.e^{-2 d} \mathcal{R}\right|_{\tilde{\partial}=0}$ defines the action

$$
S_{*}^{\prime}=\int d x \sqrt{-g} e^{-2 \phi}\left[R+4 \square \phi-4(\partial \phi)^{2}-\frac{1}{12} H^{2}\right],
$$

which differs by integrals of total derivatives from the familiar action $S_{*}$ in (1.6). Moreover, one quickly verifies that the dilaton equation of motion arises from $S_{*}^{\prime}$ by solely varying the exponential $\exp (-2 \phi)$. This is, of course, the same equation of motion that follows from $S_{*}$.

The dilaton equation of motion of $S_{*}$ can be expressed as a linear combination of graviton and dilaton beta-functions $\beta_{i j}^{g}$ and $\beta^{\phi}$ of the associated two-dimensional sigma model. Using the formulae in $\S 3.7$ of [18], and $\alpha^{\prime}=1$ we readily find that

$$
\beta^{d} \equiv \beta^{\phi}-\frac{1}{4} g^{i j} \beta_{i j}^{g}=-\frac{1}{4}\left(R+4 \square \phi-4(\partial \phi)^{2}-\frac{1}{12} H^{2}\right) .
$$

Since $e^{-2 d}=\sqrt{-g} e^{-2 \phi}$, it follows that $\beta^{d}$, as the notation suggests, can be thought as the beta function for the T-duality invariant dilaton $d$. Thus, in $S_{*}^{\prime}$ the Lagrangian is proportional to the beta function of the dilaton $d$. Indeed, $\beta^{d}$ was used in [17] to write the spacetime action as $\int \sqrt{-g} e^{-2 \phi} \beta^{d}$. Let us note that, in contrast, the integrand of $S_{*}$ is not proportional to a linear combination of beta functions. 


\section{$4.3 \quad$ T-duality and gauge invariance}

In this section we examine the relationship between the $O(D, D)$ duality symmetry and the gauge symmetry of the theory we have constructed. We first consider the theory in $\mathbb{R}^{2 D}$ and focus on the infinitesimal form of the $O(D, D)$ transformations. We write the group element as $\mathbf{1}+T$, with $T$ in the Lie algebra, and use the basis

$$
\left(\begin{array}{cc}
h & 0 \\
0 & -h^{t}
\end{array}\right), \quad\left(\begin{array}{ll}
0 & e \\
0 & 0
\end{array}\right), \quad\left(\begin{array}{ll}
0 & 0 \\
f & 0
\end{array}\right)
$$

where $h$ is an arbitrary $D \times D$ matrix, while $e$ and $f$ are antisymmetric $D \times D$ matrices 3 From (2.60), the corresponding infinitesimal $O(D, D)$ transformations are given by

$$
\begin{array}{rlrl}
h: & & \mathcal{E}^{\prime}\left(X^{\prime}\right)=\mathcal{E}(X)+\mathcal{E}(X) h^{t}+h \mathcal{E}(X), \\
e: & \mathcal{E}^{\prime}\left(X^{\prime}\right)=\mathcal{E}(X)+e, \\
f: & \mathcal{E}^{\prime}\left(X^{\prime}\right)=\mathcal{E}(X)-\mathcal{E}(X) f \mathcal{E}(X) .
\end{array}
$$

If we write

$$
X^{\prime M}=X^{M}-\xi^{M}(X)
$$

these transformations can be written as variations $\delta \mathcal{E}(X) \equiv \mathcal{E}^{\prime}(X)-\mathcal{E}(X)$ that take the form

$$
\begin{aligned}
\delta_{h} \mathcal{E} & =\xi^{M} \partial_{M} \mathcal{E}+\mathcal{E} h^{t}+h \mathcal{E}, \\
\delta_{e} \mathcal{E} & =\xi^{M} \partial_{M} \mathcal{E}+e \\
\delta_{f} \mathcal{E} & =\xi^{M} \partial_{M} \mathcal{E}-\mathcal{E} f \mathcal{E} .
\end{aligned}
$$

The strong constraint implies that the fields are restricted to a null subspace $N$. In the case in which the fields depend just on $x$ and are independent of $\tilde{x}$, the constraint is satisfied by parameters that depend just on $x$ and are independent of $\tilde{x}$. All other choices of $N$ are related to this by an $O(D, D)$ transformation, so it is sufficient to consider this case. Choosing parameters

$$
\tilde{\xi}_{i}=-\frac{1}{2} e_{i j} x^{j}, \quad \xi^{i}=x^{j} h_{j}^{i}
$$

the gauge transformation (2.31) take the form

$$
\delta_{\xi} \mathcal{E}_{i j}=\xi^{k} \partial_{k} \mathcal{E}_{i j}+\left(\mathcal{E} h^{t}+h \mathcal{E}\right)_{i j}+e_{i j}
$$

We see that the $O(D, D)$ transformations (4.43) and (4.44) arise from gauge transformations. This is the expected result that the infinitesimal $G L(D, \mathbb{R})$ transformations with parameter $h$ arise from diffeomorphisms and the constant shifts of the $b$-field with parameter $e$ from anti-symmetric tensor gauge transformations. Note that exponentiating the $h$ transformations only generates a subgroup of $G L(D, \mathbb{R})$ (with positive determinant). However, the full $G L(D, \mathbb{R})$ symmetry in fact arises from

\footnotetext{
${ }^{3}$ We only use $e, f, h$ in this way in this subsection, and this should not be confused with the uses of $e, f, h$ elsewhere in the paper.
} 
diffeomorphisms of the spacetime with coordinates $x^{i}$. Thus, the $G L(D, \mathbb{R}) \ltimes \mathbb{R}^{D(D-1) / 2}$ 'geometric subgroup' of $O(D, D)$ consisting of $G L(D, \mathbb{R})$ transformations plus $b$-shifts arises from gauge transformations.

Consider now the remaining infinitesimal $O(D, D)$ transformations with constant parameter $f$. A natural ansatz is to consider gauge transformations with parameters

$$
\tilde{\xi}_{i}=0, \quad \xi^{i}=-\frac{1}{2} f^{i j} \tilde{x}_{j} .
$$

The constraint requires that $\partial^{M} \xi^{i} \partial_{M} A=0$ for all fields $A$, which here implies

$$
f^{i j} \partial_{j} A=0
$$

for all fields $A$. In general, the fields $\mathcal{E}, d$ will depend on all the coordinates $x^{i}$ and (4.49) will have no solutions, so that the $f$-symmetries do not arise from gauge transformations. If the theory, however, is truncated to a subsector in which the fields are also independent of some of the coordinates $x^{i}$, then some of the $O(D, D)$ transformations generated by $f^{i j}$ do arise from gauge symmetries. Suppose then that the $D$ coordinates $x^{i}$ are split into $d$ coordinates $x^{a}$ and $D-d$ coordinates $x^{\mu}$, with a corresponding split $\tilde{x}_{i}=\left(\tilde{x}_{\mu}, \tilde{x}_{a}\right)$, and consider the truncation to the subsector in which the fields are independent of the $d$ coordinates $x^{a}$ as well as independent of $\tilde{x}_{i}$. The fields $\mathcal{E}_{i j}$ and $d$ in the truncated sector then depend on $x^{\mu}$ only, so that $\partial_{a} A=0$ for all fields $A$. We take the only non-vanishing components of $f^{i j}$ to be $f^{a b}$, giving gauge parameters

$$
\tilde{\xi}_{i}=0, \quad \xi^{\mu}=0, \quad \xi^{a}=-\frac{1}{2} f^{a b} \tilde{x}_{b} .
$$

The constraint (4.49) is now satisfied as $\partial_{a} A=0$, and so (4.50) leads to allowed gauge transformations in the truncated subsector. They read

$$
\delta_{\xi} \mathcal{E}_{i j}=-(\mathcal{E} f \mathcal{E})_{i j}=-\mathcal{E}_{i a} f^{a b} \mathcal{E}_{b j}
$$

which agrees with (4.45) using the fact that $\xi^{M} \partial_{M} \mathcal{E}_{i j}=0$ for these parameters.

The parameters $h_{a}{ }^{b}$ are generators of a $G L(d, \mathbb{R})$ symmetry arising from diffeomorphisms acting on the $x^{a}$, while the transformations generated by $e_{a b}$ arise from $b$-field gauge transformations. Then the parameters $h_{a}{ }^{b}, e_{a b}, f^{a b}$ are generators of an $O(d, d)$ subgroup of the $O(D, D)$ symmetry. These infinitesimal $O(d, d)$ symmetries all arise from infinitesimal gauge transformations of the truncated theory, as we have seen. Thus the theory truncated to be independent of the $x^{a}$ (as well as $\tilde{x}_{i}$ ) has an $O(d, d)$ symmetry that arises from gauge transformations. Strictly speaking, we have only shown this for the subgroup that arises from exponentiating infinitesimal generators. The remaining symmetries in $O(d, d)$ might be thought of as large gauge transformations - to understand this better would require knowledge of the finite form of the gauge symmetries. Note that the vector fields $\frac{\partial}{\partial x^{a}}$ can be regarded as $d$ commuting Killing vectors.

We return now to the general case (without isometries), where the $h$ and $e$ transformations generate a $G L(D, \mathbb{R}) \ltimes \mathbb{R}^{D(D-1) / 2}$ subgroup of the $O(D, D)$ symmetry that can arise from gauge transformations. If $d$ of the $D$ coordinates are compactified on a torus the spacetime is $\mathbb{R}^{n-1,1} \times T^{d}$ and the $O(d, d)$ 
subgroup of the $O(D, D)$ symmetry acting on the toroidal directions is broken to $O(d, d ; \mathbb{Z})$ by the periodic boundary conditions. Then a $G L(d, \mathbb{Z}) \ltimes \mathbb{Z}^{d(d-1) / 2}$ subgroup of $O(d, d ; \mathbb{Z})$ arises through (large) gauge transformations. Indeed, $G L(d, \mathbb{Z})$ is the familiar mapping class group of large diffeomorphisms of $T^{d}$. In Kaluza-Klein compactification, one considers the truncation of the theory to the subsector of fields that are independent of $d$ toroidal coordinates. As there is no dependence on the $d$ toroidal coordinates, this is the same as the truncation of the theory on $\mathbb{R}^{n} \times \mathbb{R}^{d}$ to fields on $\mathbb{R}^{n}$ considered above. This truncated theory has a continuous $O(d, d)$ symmetry and we have already seen that this is generated by gauge transformations on $\mathbb{R}^{n} \times \mathbb{R}^{d}$. However, these are not gauge transformations of the theory on $\mathbb{R}^{n} \times T^{d}$; at most the discrete subgroup $O(d, d ; \mathbb{Z})$ can arise from gauge symmetries of the theory on $\mathbb{R}^{n} \times T^{d}$.

The previous discussion sheds an interesting light on the symmetries of Kaluza-Klein theory. Any gravity theory reduced on a $d$-torus has a $G L(d, \mathbb{R})$ symmetry [26]. The truncated theory is the same as the theory in which the internal torus is replaced by $\mathbb{R}^{d}$ with no dependence on the extra $d$ coordinates, and the $G L(d, \mathbb{R})$ symmetry is then a remnant of the ordinary diffeomorphism symmetry of $\mathbb{R}^{d}$. Only a discrete subgroup $G L(d, \mathbb{Z})$ is inherited from diffeomorphisms of the internal space $T^{d}$. Similarly, the continuous shift symmetry of the internal two-form is a remnant of the abelian gauge symmetry of the Kalb-Ramond field on $\mathbb{R}^{d}$, and again only a discrete subgroup is properly a consequence of the gauge symmetry on $T^{d}$. The most interesting symmetries are the non-linear transformations (denoted by $f$ in (4.40) $)$, which complete the $G L(d)$ and shift symmetries to the duality group $O(d, d)$. These do not have a higher-dimensional explanation in the usual formulation and are often referred to as 'hidden symmetries', see, e.g., [25, 27] and references therein. These are now seen as arising from gauge transformations of the double field theory. For the Kaluza-Klein theory on $\mathbb{R}^{n} \times T^{d}$, the $O(d, d ; \mathbb{Z})$ transformations are precisely the so-called Buscher rules [30, 31]. We should stress, however, that at best only the discrete subgroup $O(d, d ; \mathbb{Z})$ arises from gauge transformation of the theory on $\mathbb{R}^{n} \times T^{d}$, and to complete the proof of this would require knowledge of the so far unknown finite form of the gauge transformations.

\section{Concluding remarks}

In this paper, we have extended spacetime by introducing extra coordinates $\tilde{x}$ and constructed a theory in the doubled space that is $O(D, D)$ invariant and which reduces to the conventional theory of Einstein gravity plus antisymmetric tensor plus dilaton when the fields are restricted to be independent of the extra coordinates $\tilde{x}$. As such, it could be viewed as an $O(D, D)$ covariantization of the usual theory in which a dependence on dual coordinates $\tilde{x}$ is introduced and an $O(D, D)$ invariant constraint that effectively removes this dependence is imposed.

The theory has a remarkable $O(D, D)$ duality symmetry in flat space, broken to a subgroup containing the T-duality group $O(d, d ; \mathbb{Z})$ when $d$ dimensions are compactified to form a $d$-torus. Not only are $g$ and $b$ mixed by this symmetry, but the diffeomorphism and antisymmetric tensor gauge

parameters are also rotated into each other. The T-duality transformations are a generalisation of the usual Buscher transformations to the case in which the fields can have arbitrary dependence on the 
toroidal coordinates.

The full double field theory that arises from the massless sector of closed string theory is expected to have many novel features, as we have discussed elsewhere [2, 3]. Our construction here can be viewed as a reduction of the complete double field theory using our strong constraint, or equivalently restricting to a totally null subspace. We view our construction as an important step towards the elucidation of the full theory.

An important feature of (1.1) is the background independence. Our construction has been based on a flat doubled space with possible toroidal identifications, but it is interesting to ask whether it could have a wider validity to more general double spaces. The action uses double coordinates $X^{M}$, together with a way of splitting the $2 D$ vector indices into two sets of indices, so that a vector decomposes as $V^{M}=\left(\tilde{v}_{i}, v^{i}\right)$, allowing quantities with $i, j$ indices such as $\mathcal{E}_{i j}$ to be defined. This index splitting requires that the double space admit an almost local product structure. The constraint uses an $O(D, D)$ metric $\eta$. Here it is a constant metric and the existence of this flat metric restricts the double space to be a flat space, given by $\mathbb{R}^{2 D}$ or obtained from it by suitable identifications. It would be interesting to seek generalisations to more general spaces in which the constraint involves a non-flat metric of signature $(D, D)$. For example, there is some evidence that doubled groups should play a role in formulating less trivial string backgrounds in this context [19, 20]. It would be of considerable interest to seek an extension of this formalism that was applicable to spaces with a torus fibration or to T-folds; in both cases, it is expected that double geometry should play a useful role [21, 22.

It is interesting to discuss the relation of our work to that in non-symmetric gravity theories [16]. A geometric action for a theory based on a non-symmetric tensor $\mathcal{E}$ is usually taken to involve the use of $\mathcal{E}$ and its inverse to define curvatures and torsions and contract indices. A theory of the type $R+H^{2}+\ldots$ that appears in string theory requires an infinite number of geometrical terms and is thus not considered natural in that framework [16]. Such actions generally have a problem: if $\mathcal{E}$ is used to contract indices, the antisymmetric tensor $b$ appears without derivatives, which violates gauge invariance. Our theory avoids this problem by using $g=\frac{1}{2}\left(\mathcal{E}+\mathcal{E}^{t}\right)$ to contract indices.

The fields $g$ and $b$ transform independently under diffeomorphisms, so that $\mathcal{E}=g+b$ transforms reducibly. In contrast, the double field theory constructed in this paper admits an enlarged gauge symmetry, under which the symmetric and antisymmetric part of $\mathcal{E}_{i j}$ transform into each other. Moreover, the $O(D, D)$ symmetry acts irreducibly on $\mathcal{E}$. Therefore, the action (1.1) can be seen as a geometrical unification of metric and 2-form, even though it would not be considered geometrical in the sense of [16] because we contract indices using the inverse metric $g^{i j}$ instead of the inverse of $\mathcal{E}_{i j}$. For us, using $g^{i j}$ is natural - it is an $O(D, D)$ tensor and does not involve $b_{i j}$. If there had been inverses of $\mathcal{E}_{i j}$ appearing without derivatives, there would be terms with undifferentiated $b_{i j}$ fields which could not be gauge invariant. A gauge invariant action cannot be constructed using $\mathcal{E}_{i j}$ alone, and introducing the dilaton $d$ is essential for gauge invariance.

There remain a number of issues to be investigated. We have made some preliminary remarks about a possible $O(D, D)$ geometry, but it is important to understand fully the geometry underlying our theory, its symmetries, and the 'scalar curvature' that appears in the action. One geometry has been proposed in this context by Siegel [5], and generalised geometry [7, 8, 9, 10, 11] might provide 
a useful geometric framework. It would be interesting to understand whether our construction fits within either of these geometric structures.

There are a number of natural directions in which this research might be developed. It would be interesting to extend the field content to incorporate the RR $p$-form gauge fields of type II supergravity. Another interesting generalisation would be to try to go beyond the T-duality group $O(D, D)$ and 'geometrize' the exceptional U-duality groups that arise in compactifications of the full type II theory. In [21], it was proposed that the double torus representing momentum and string winding modes had a natural generalisation to a higher torus representing brane wrapping modes as well, with a natural action of the U-duality group on it [32]. For toroidal compactifications to 4 dimensions, for example, the appropriate torus is 56-dimensional with a natural action of the U-duality group $E_{7}(\mathbb{Z})[27,21$. Generalisations of generalised geometry were proposed in [23] and developed in [24] in which $O(D, D)$ was replaced by U-duality groups; see also [33]. Such geometries may play a key role in U-duality generalisations of our present work.

\section{Acknowledgments}

We are happy to acknowledge helpful correspondence with Marco Gualtieri and discussions with Matthew Headrick, Hermann Nicolai, Ashoke Sen, and Dan Waldram. This work is supported by the U.S. Department of Energy (DoE) under the cooperative research agreement DE-FG02-05ER41360. The work of $\mathrm{OH}$ is supported by the DFG - The German Science Foundation.

\section{A Derivation of the background independent gauge transformations}

In this appendix, we give some of the details of the derivation of the background independent gauge transformations given in section [2.2, using results from [3].

Recall that the full background independent field is

$$
\mathcal{E}_{i j} \equiv E_{i j}+\check{e}_{i j}
$$

with a constant background $E_{i j}$ and a fluctuation field $\check{e}_{i j}$ related to the double field theory fluctuation $e_{i j}$ by the matrix relation

$$
\check{e}=F(e) e=e F(e)=f(e)
$$

where the function $F(e)$ is given by

$$
F(e) \equiv\left(1-\frac{1}{2} e\right)^{-1}
$$

It is an immediate consequence of the definitions that $\check{e}$ and $e$ commute:

$$
\check{e} e=e \check{e} .
$$

It follows that

$$
e=\left(1-\frac{1}{2} e\right) \check{e}=\check{e}\left(1-\frac{1}{2} e\right)
$$


The above leads to

$$
\check{e}-e=\frac{1}{2} e \check{e}=\frac{1}{2} \check{e} e,
$$

and one readily verifies that

$$
\left(1+\frac{1}{2} \check{e}\right)\left(1-\frac{1}{2} e\right)=1 .
$$

Varying (A.6) and using (A.7) we find a relation between arbitrary variations,

$$
\delta e=\left(1-\frac{1}{2} e\right) \delta \check{e}\left(1-\frac{1}{2} e\right) .
$$

Then for any variation or derivative

$$
\delta \mathcal{E}=\delta \check{e}=F \delta e F .
$$

It will be useful to split the variation in (2.20) into a transport variation $\delta_{T}$ given by

$$
\delta_{T} e_{i j}=\frac{1}{2}(\lambda \cdot D+\bar{\lambda} \cdot \bar{D}) e_{i j},
$$

and the rest $\delta_{R}$, so that $\delta e=\delta_{T} e+\delta_{R} e$. Then we find

$$
\delta_{T} \mathcal{E}=F \delta_{T} e F=\frac{1}{2} F[(\lambda \cdot D+\bar{\lambda} \cdot \bar{D}) e] F=\frac{1}{2}(\lambda \cdot D+\bar{\lambda} \cdot \bar{D}) \mathcal{E},
$$

using that (A.9) implies $D \mathcal{E}=F(D e) F$, etc. Thus,

$$
\delta_{T} \mathcal{E}_{i j}=\frac{1}{2}(\lambda \cdot D+\bar{\lambda} \cdot \bar{D}) \mathcal{E}_{i j} .
$$

Next, we consider

$$
\delta_{R} e_{i j}=\hat{D}_{i} \bar{\lambda}_{j}+\hat{\bar{D}}_{j} \lambda_{i}+\frac{1}{2}\left(\hat{D}_{i} \lambda^{k}\right) e_{k j}+\frac{1}{2} e_{i k} \hat{\bar{D}}_{j} \bar{\lambda}^{k}
$$

which implies

$$
\delta_{R} \mathcal{E}_{k l}=F_{k i}\left[\hat{D}_{i} \bar{\lambda}_{j}+\hat{\bar{D}}_{j} \lambda_{i}+\frac{1}{2}\left(\hat{D}_{i} \lambda^{m}\right) e_{m j}+\frac{1}{2} e_{i m} \hat{\bar{D}}_{j} \bar{\lambda}^{m}\right] F_{j l}
$$

We now claim that the derivatives

$$
\mathcal{D}_{i} \equiv F_{i j} \hat{D}_{j}, \quad \overline{\mathcal{D}}_{i} \equiv F_{j i} \hat{\bar{D}}_{j},
$$

agree with the previous definitions, as they are given by

$$
\mathcal{D}_{i}=\partial_{i}-\mathcal{E}_{i k} \tilde{\partial}^{k}, \quad \overline{\mathcal{D}}_{i}=\partial_{i}+\mathcal{E}_{k i} \tilde{\partial}^{k}
$$

This is readily confirmed. For example,

$$
\begin{aligned}
\mathcal{D} & =F\left(D-\frac{1}{2} e \bar{D}\right)=F D-\frac{1}{2} f \bar{D}=\left(1+\frac{1}{2} f\right) D-\frac{1}{2} f \bar{D} \\
& =D-\frac{1}{2} f(\bar{D}-D)=D-\check{e} \tilde{\partial}=\partial-\mathcal{E} \tilde{\partial}
\end{aligned}
$$


where we used $1+\frac{1}{2} f=F$. Then (A.14) can be rewritten as

$$
\delta_{R} \mathcal{E}_{k l}=F_{j l} \mathcal{D}_{k} \bar{\lambda}_{j}+F_{k i} \overline{\mathcal{D}}_{l} \lambda_{i}+\frac{1}{2}\left(\mathcal{D}_{k} \lambda^{m}\right) \check{e}_{m l}+\frac{1}{2} \check{e}_{k m} \overline{\mathcal{D}}_{l} \bar{\lambda}^{m} .
$$

Using $1+\frac{1}{2} f=F$ this can be further rewritten as

$$
\delta_{R} \mathcal{E}_{k l}=\mathcal{D}_{k} \bar{\lambda}_{l}+\overline{\mathcal{D}}_{l} \lambda_{k}+\frac{1}{2} \check{e}_{m l} \mathcal{D}_{k} \bar{\lambda}^{m}+\frac{1}{2} \check{e}_{k m} \overline{\mathcal{D}}_{l} \lambda^{m}+\frac{1}{2}\left(\mathcal{D}_{k} \lambda^{m}\right) \check{e}_{m l}+\frac{1}{2} \check{e}_{k m} \overline{\mathcal{D}}_{l} \bar{\lambda}^{m}
$$

We then infer

$$
\delta_{R} \mathcal{E}_{k l}=\mathcal{D}_{k} \bar{\lambda}_{l}+\overline{\mathcal{D}}_{l} \lambda_{k}+\frac{1}{2} \check{e}_{m l} \mathcal{D}_{k}\left(\bar{\lambda}^{m}+\lambda^{m}\right)+\frac{1}{2} \check{e}_{k m} \overline{\mathcal{D}}_{l}\left(\lambda^{m}+\bar{\lambda}^{m}\right)
$$

Next we rewrite this in terms of the gauge parameters $(\tilde{\xi}, \xi)$. First, using $\frac{1}{2}\left(\lambda^{m}+\bar{\lambda}^{m}\right)=\xi^{m}$ we have

$$
\begin{aligned}
\delta_{R} \mathcal{E}_{k l} & =\mathcal{D}_{k} \bar{\lambda}_{l}+\overline{\mathcal{D}}_{l} \lambda_{k}+\left(\mathcal{E}_{m l}-E_{m l}\right) \mathcal{D}_{k} \xi^{m}+\left(\mathcal{E}_{k m}-E_{k m}\right) \overline{\mathcal{D}}_{l} \xi^{m} \\
& =\mathcal{D}_{k}\left(\bar{\lambda}_{l}-E_{m l} \xi^{m}\right)+\overline{\mathcal{D}}_{l}\left(\lambda_{k}-E_{k m} \xi^{m}\right)+\mathcal{E}_{m l} \mathcal{D}_{k} \xi^{m}+\mathcal{E}_{k m} \overline{\mathcal{D}}_{l} \xi^{m}
\end{aligned}
$$

Using further the inverse relations (2.17) one finds

$$
\begin{aligned}
\lambda-E \xi & =-\tilde{\xi}+E \xi-E \xi=-\tilde{\xi}, \\
\bar{\lambda}-E^{t} \xi & =\tilde{\xi}+E^{t} \xi-E^{t} \xi=\tilde{\xi} .
\end{aligned}
$$

This gives the final result for the gauge transformations

$$
\delta \mathcal{E}_{i j}=\mathcal{D}_{i} \tilde{\xi}_{j}-\overline{\mathcal{D}}_{j} \tilde{\xi}_{i}+\mathcal{D}_{i} \xi^{k} \mathcal{E}_{k j}+\overline{\mathcal{D}}_{j} \xi^{k} \mathcal{E}_{i k}+\frac{1}{2}(\lambda \cdot D+\bar{\lambda} \cdot \bar{D}) \mathcal{E}_{i j}
$$

The only terms that look out of place here are those in the last term, the transport one. They are easily seen, however, to be equal to $\xi^{i} \partial_{i}+\tilde{\xi}_{i} \tilde{\partial}^{i}=\xi^{M} \partial_{M}$. Then the gauge transformation can be rewritten as

$$
\delta \mathcal{E}_{i j}=\mathcal{D}_{i} \tilde{\xi}_{j}-\overline{\mathcal{D}}_{j} \tilde{\xi}_{i}+\xi^{M} \partial_{M} \mathcal{E}_{i j}+\mathcal{D}_{i} \xi^{k} \mathcal{E}_{k j}+\overline{\mathcal{D}}_{j} \xi^{k} \mathcal{E}_{i k}
$$

which is the final form used in the main text.

\section{B Explicit check of gauge invariance}

As a warm-up we begin by checking that the action $S^{(0)}$ in (3.18) is gauge invariant under $\delta^{(0)}$. This, of course, is guaranteed to work. As explained in the main text, the strategy will be to keep track only of those transformations that do not take the form of a Lie derivative and that are therefore not guaranteed to yield terms that combine into total derivatives. For the variation $\delta^{(0)} \mathcal{L}^{(0)}$ it is therefore sufficient to focus on the terms that involve a partial derivative. For the variations of the partial derivatives we use

$$
\begin{aligned}
\delta\left(\partial_{p} g_{k l}\right) & =\mathcal{L}_{\xi}\left(\partial_{p} g_{k l}\right)+\partial_{p} \partial_{k} \xi^{q} g_{l q}+\partial_{p} \partial_{l} \xi^{q} g_{k q} \\
\delta\left(\partial_{i} d\right) & =\mathcal{L}_{\xi}\left(\partial_{i} d\right)-\frac{1}{2} \partial_{i} \partial_{j} \xi^{j}
\end{aligned}
$$


where the Lie derivative $\mathcal{L}_{\xi}$ represents the covariant terms. We do not have to vary the $H^{2}$ term because the 3 -form field strength transforms covariantly. The variation reads

$$
\begin{aligned}
\delta \mathcal{L}^{(0)}=e^{-2 d}[ & -g^{i k} \partial^{p} g_{k l} \partial_{p} \partial_{i} \xi^{l}+\partial^{j} g_{i k} \partial^{i} \partial_{j} \xi^{k} \\
& +\partial_{q} g_{i k} g^{k l} \partial^{i} \partial_{l} \xi^{q}-\partial^{i} \partial_{k} \xi^{k} \partial^{j} g_{i j} \\
& \left.+2 \partial^{i} d \partial_{j} \partial_{i} \xi^{j}+2 \partial_{i} d g^{k l} \partial_{k} \partial_{l} \xi^{i}-4 \partial^{i} d \partial_{i} \partial_{j} \xi^{j}\right] .
\end{aligned}
$$

Commuting partial derivatives and relabeling the indices, we see that the two terms in the first line cancel and that two terms in the last line combine. Since ultimately we have to partially integrate it is convenient to rewrite terms in the last line as derivatives on $e^{-2 d}$. In total we get

$$
\begin{aligned}
\delta \mathcal{L}^{(0)}= & e^{-2 d}\left(g^{k l} g^{i j} \partial_{q} g_{i k} \partial_{j} \partial_{l} \xi^{q}-g^{i j} g^{k l} \partial_{j} \partial_{q} \xi^{q} \partial_{k} g_{i l}\right) \\
& +g^{i j} \partial_{j}\left(e^{-2 d}\right) \partial_{i} \partial_{q} \xi^{q}-g^{k l} \partial_{i}\left(e^{-2 d}\right) \partial_{k} \partial_{l} \xi^{i}
\end{aligned}
$$

Here all metrics are written explicitly. If we use $g^{k l} g^{i j} \partial_{q} g_{i k}=-\partial_{q} g^{l j}$, etc., the terms in the first line can be written up to total derivatives as

$$
\begin{aligned}
-e^{-2 d} \partial_{q} g^{l j} \partial_{j} \partial_{l} \xi^{q} & =\partial_{q}\left(e^{-2 d}\right) g^{j l} \partial_{j} \partial_{l} \xi^{q}+e^{-2 d} g^{l j} \partial_{l} \partial_{j} \partial_{q} \xi^{q} \\
e^{-2 d} \partial_{k} g^{j k} \partial_{j} \partial_{q} \xi^{q} & =-\partial_{k}\left(e^{-2 d}\right) g^{j k} \partial_{j} \partial_{q} \xi^{q}-e^{-2 d} g^{j k} \partial_{k} \partial_{j} \partial_{q} \xi^{q} .
\end{aligned}
$$

The terms with $\partial^{3} \xi$ cancel each other, while the terms with $\partial\left(e^{-2 d}\right)$ cancel against the terms in the second line of (B.3). Thus, as expected, $\delta \mathcal{L}^{(0)}=0$ follows.

We now turn to the proof of full gauge invariance using the derivative expansion (3.15). As we stated in the main text, it is sufficient to verify the third condition in (3.32). For this, it is convenient to simplify the expression for $S^{(1)}$ given in (3.27). Upon relabeling of indices, the terms cubic in $b$ can be rewritten in a form that is proportional to the invariant field strength $H_{i j k}$. To identify terms unambiguously we rewrite every term with a single dilaton derivative term according to $e^{-2 d} \partial_{i} d=-\frac{1}{2} \partial_{i}\left(e^{-2 d}\right)$ and then partially integrate in order to move the derivative away from the dilaton. This leads to further simplifications and the final form of $\mathcal{L}^{(1)}$ reads

$$
\begin{aligned}
\mathcal{L}^{(1)}=e^{-2 d}[ & \frac{1}{2} g^{i k} g^{j l} g^{p q}\left(b_{i r} \tilde{\partial}^{r} b_{j p} H_{k l q}+b_{p r} \tilde{\partial}^{r} g_{k l} \partial_{q} g_{i j}-2 b_{l r} \tilde{\partial}^{r} g_{i p} \partial_{k} g_{j q}\right) \\
& -g^{i k} g^{p q} \tilde{\partial}^{j} b_{i p} \partial_{k} g_{j q}+2 b_{i r} \partial_{j} \tilde{\partial}^{r} g^{i j}+2 \tilde{\partial}^{k} b_{i k} \partial_{j} g^{i j} \\
& \left.+2 g^{i j} \partial_{i} \tilde{\partial}^{k} b_{j k}+\tilde{\partial}^{k} g^{i j} \partial_{i} b_{j k}-8 g^{i j} b_{i k} \tilde{\partial}^{k} d \partial_{j} d\right]+(\mathrm{td}) .
\end{aligned}
$$

We do the same with the single dilaton derivative in $\mathcal{L}^{(0)}$,

$$
\begin{aligned}
\mathcal{L}^{(0)}=e^{-2 d} & -\frac{1}{4} g^{i k} g^{j l} \partial^{p} g_{k l} \partial_{p} g_{i j}+\frac{1}{2} g^{k l} \partial^{j} g_{i k} \partial^{i} g_{j l} \\
& \left.-\partial_{i} \partial_{j} g^{i j}+4 \partial^{i} d \partial_{i} d-\frac{1}{12} H^{i j k} H_{i j k}\right)+(\mathrm{td}) .
\end{aligned}
$$

For simplicity we begin the check of gauge invariance with only $\tilde{\xi}_{i}$ non-zero. To vary $\mathcal{L}^{(1)}$ we use $\delta^{(0)} b_{i j}=\partial_{i} \tilde{\xi}_{j}-\partial_{j} \tilde{\xi}_{i}$, while $\delta^{(0)}$ is trivial on all other fields. Thus we have to vary only the $b$ 's inside 
(B.5). For the variation of $\mathcal{L}^{(0)}$ under $\delta^{(1)}$ we use

$$
\delta^{(1)} g_{i j}=\mathcal{L}_{\tilde{\xi}} g_{i j}, \quad \delta^{(1)} b_{i j}=\mathcal{L}_{\tilde{\xi}} b_{i j}, \quad \delta^{(1)} d=\tilde{\xi}_{i} \tilde{\partial}^{i} d-\frac{1}{2} \tilde{\partial}^{i} \tilde{\xi}_{i}
$$

where $\mathcal{L}_{\tilde{\xi}}$ denote the Lie derivative with respect to the 'dual diffeomorphism' parameter $\tilde{\xi}_{i}$. Moreover, the dilaton transforms as a density under these dual diffeomorphisms. Again, it is guaranteed that all variations that are covariant in this sense combine into the total derivative $\tilde{\partial}^{i}\left(\tilde{\xi}_{i} \mathcal{L}\right)$. Since we have an integration over $d \tilde{x}$, these total derivatives can be ignored. We only have to work out the variations that are non-covariant (in the dual sense), and the only source for those terms are partial derivatives. Even the $H$ field is not covariant anymore under the dual diffeomorphisms:

$$
\delta_{\tilde{\xi}}^{(1)} H_{i j k}=\mathcal{L}_{\tilde{\xi}} H_{i j k}+3 \tilde{\partial}^{p} \tilde{\xi}_{[i} \partial_{|p|} b_{j k]}+3 \partial_{[i} \tilde{\xi}_{|p|} \tilde{\partial}^{p} b_{j k]}+6 \partial_{[i} \tilde{\partial}^{p} \tilde{\xi}_{j} b_{k] p}
$$

For the partial derivatives of $g_{i j}, b_{i j}$, and $d$ one finds

$$
\begin{aligned}
\delta_{\tilde{\xi}}^{(1)}\left(\partial_{i} g_{j k}\right) & =\mathcal{L}_{\tilde{\xi}}\left(\partial_{i} g_{j k}\right)+\tilde{\partial}^{p} \tilde{\xi}_{i} \partial_{p} g_{j k}+\partial_{i} \tilde{\xi}_{p} \tilde{\partial}^{p} g_{j k}-2 \partial_{i} \tilde{\partial}^{p} \tilde{\xi}_{(j} g_{k) p}, \\
\delta_{\tilde{\xi}}^{(1)}\left(\partial_{i} b_{j k}\right) & =\mathcal{L}_{\tilde{\xi}}\left(\partial_{i} b_{j k}\right)+\tilde{\partial}^{p} \tilde{\xi}_{i} \partial_{p} b_{j k}+\partial_{i} \tilde{\xi}_{p} \tilde{\partial}^{p} b_{j k}+2 \partial_{i} \tilde{\partial}^{p} \tilde{\xi}_{[j} b_{k] p}, \\
\delta_{\tilde{\xi}}^{(1)}\left(\partial_{i} g^{j k}\right) & =\mathcal{L}_{\tilde{\xi}}\left(\partial_{i} g^{j k}\right)+\tilde{\partial}^{p} \tilde{\xi}_{i} \partial_{p} g^{j k}+\partial_{i} \tilde{\xi}_{p} \tilde{\partial}^{p} g^{j k}+2 g^{p(j} \partial_{i} \tilde{\partial}^{k)} \tilde{\xi}_{p}, \\
\delta_{\tilde{\xi}}^{(1)}\left(\partial_{i} d\right) & =\mathcal{L}_{\tilde{\xi}}\left(\partial_{i} d\right)+\tilde{\partial}^{k} \tilde{\xi}_{i} \partial_{k} d+\partial_{i} \tilde{\xi}_{k} \tilde{\partial}^{k} d-\frac{1}{2} \partial_{i} \tilde{\partial}^{k} \tilde{\xi}_{k} .
\end{aligned}
$$

Finally, we need the variation of the double (partial) divergence of $g^{i j}$,

$$
\delta_{\tilde{\xi}}^{(1)}\left(\partial_{i} \partial_{j} g^{i j}\right)=\mathcal{L}_{\tilde{\xi}}\left(\partial_{i} \partial_{j} g^{i j}\right)+2 \partial_{i} \partial_{j} g^{i p} \tilde{\partial}^{j} \tilde{\xi}_{p}+2 \partial_{i} \tilde{\xi}_{p} \tilde{\partial}^{p} \partial_{j} g^{i j}+2 \partial_{j} g^{i p} \partial_{i} \tilde{\partial}^{j} \tilde{\xi}_{p}+\partial_{i} \partial_{j} \tilde{\xi}_{p} \tilde{\partial}^{p} g^{i j}
$$

It is now straightforward to vary the partial derivatives in $\mathcal{L}^{(0)}$ by the non-covariant terms given here and the $b_{i j}$ in $\mathcal{L}^{(1)}$ according to the standard abelian 2-form transformations. To see how this gauge invariance works let us illustrate the cancellation for the terms quadratic in derivatives on $d$. Varying these terms in $\mathcal{L}^{(0)}$ and $\mathcal{L}^{(1)}$ one finds

$$
\begin{aligned}
\delta^{(0)} \mathcal{L}^{(1)}+\delta^{(1)} \mathcal{L}^{(0)} & =\delta^{(0)}\left(-8 e^{-2 d} g^{i j} b_{i k} \tilde{\partial}^{k} d \partial_{j} d\right)+\delta^{(1)}\left(4 e^{-2 d} g^{i j} \partial_{i} d \partial_{j} d\right) \\
& =8 e^{-2 d} \partial^{i} d\left(\partial_{k} \tilde{\xi}_{i} \tilde{\partial}^{k} d-\partial_{i} \tilde{\xi}_{k} \tilde{\partial}^{k} d+\tilde{\partial}^{k} \tilde{\xi}_{i} \partial_{k} d+\partial_{i} \tilde{\xi}_{k} \tilde{\partial}^{k} d-\frac{1}{2} \partial_{i} \tilde{\partial}^{j} \tilde{\xi}_{j}\right) \\
& =8 e^{-2 d} \partial^{i} d\left(\partial_{k} \tilde{\xi}_{i} \tilde{\partial}^{k} d+\tilde{\partial}^{k} \tilde{\xi}_{i} \partial_{k} d\right)-4 e^{-2 d} \partial^{i} d \partial_{i} \tilde{\partial}^{k} \tilde{\xi}_{k} \\
& =-2 e^{-2 d}\left(\partial_{j} g^{i j} \partial_{i} \tilde{\partial}^{k} \tilde{\xi}_{k}+g^{i j} \partial_{i} \partial_{j} \tilde{\partial}^{k} \tilde{\xi}_{k}\right),
\end{aligned}
$$

where in the last equation we used the constraint and performed a partial integration. As stated, all terms quadratic in $d$ have cancelled. The remaining structures cancel against other contributions. Indeed, one may check that all remaining variations cancel without performing partial integrations since we have fixed the possible total-derivative ambiguity by moving all derivatives away from the dilaton. This proves full gauge invariance under $\tilde{\xi}_{i}$. 
Let us now turn to gauge invariance under $\xi^{i}$. As before, we split $\mathcal{E}_{i j}=g_{i j}+b_{i j}$. With only $\xi^{i}$ non-vanishing $\delta^{(0)}$ takes the form of the standard diffeomorphism symmetry,

$$
\delta_{\xi}^{(0)} g_{i j}=\mathcal{L}_{\xi} g_{i j}, \quad \delta_{\xi}^{(0)} b_{i j}=\mathcal{L}_{\xi} b_{i j}, \quad \delta_{\xi}^{(0)} d=\xi^{i} \partial_{i} d-\frac{1}{2} \partial_{i} \xi^{i},
$$

with the dilaton transforming as a density. Thus, in $S^{(1)}$ we only have to vary the terms that involve partial derivatives and therefore transform non-covariantly. For this we use (B.1) together with

$$
\begin{aligned}
\delta_{\xi}^{(0)}\left(\tilde{\partial}^{r} b_{j p}\right)= & \mathcal{L}_{\xi}\left(\tilde{\partial}^{r} b_{j p}\right)+\tilde{\partial}^{k} b_{j p} \partial_{k} \xi^{r}+\tilde{\partial}^{r} \xi^{k} \partial_{k} b_{j p}-2 \tilde{\partial}^{r} \partial_{[j} \xi^{k} b_{p] k}, \\
\delta_{\xi}^{(0)}\left(\tilde{\partial}^{k} g^{i j}\right)= & \mathcal{L}_{\xi}\left(\tilde{\partial}^{k} g^{i j}\right)+\tilde{\partial}^{p} g^{i j} \partial_{p} \xi^{k}+\tilde{\partial}^{k} \xi^{p} \partial_{p} g^{i j}-2 \tilde{\partial}^{k} \partial_{p} \xi^{(i} g^{j) p}, \\
\delta_{\xi}^{(0)}\left(\tilde{\partial}^{k} d\right)= & \mathcal{L}_{\xi}\left(\tilde{\partial}^{k} d\right)+\tilde{\partial}^{p} d \partial_{p} \xi^{k}+\tilde{\partial}^{k} \xi^{j} \partial_{j} d-\frac{1}{2} \tilde{\partial}^{k} \partial_{j} \xi^{j}, \\
\delta_{\xi}^{(0)}\left(\tilde{\partial}^{r} g_{k l}\right)= & \mathcal{L}_{\xi}\left(\tilde{\partial}^{r} g_{k l}\right)+\tilde{\partial}^{p} g_{k l} \partial_{p} \xi^{r}+\tilde{\partial}^{r} \xi^{p} \partial_{p} g_{k l}+2 \tilde{\partial}^{r} \partial_{(k} \xi^{p} g_{l) p}, \\
\delta_{\xi}^{(0)}\left(\partial_{i} b_{j k}\right)= & \mathcal{L}_{\xi}\left(\partial_{i} b_{j k}\right)-2 \partial_{i} \partial_{[j} \xi^{p} b_{k] p}, \\
\delta_{\xi}^{(0)}\left(\tilde{\partial}^{r} \partial_{j} g^{i j}\right)= & \mathcal{L}_{\xi}\left(\tilde{\partial}^{r} \partial_{j} g^{i j}\right)+\tilde{\partial}^{p} \partial_{j} g^{i j} \partial_{p} \xi^{r}+\tilde{\partial}^{r} \xi^{p} \partial_{p} \partial_{j} g^{i j}-\partial_{j} g^{p j} \tilde{\partial}^{r} \partial_{p} \xi^{i} \\
& -\tilde{\partial}^{r} g^{p j} \partial_{j} \partial_{p} \xi^{i}-g^{p j} \tilde{\partial}^{r} \partial_{j} \partial_{p} \xi^{i}-\tilde{\partial}^{r} g^{i p} \partial_{p} \partial_{j} \xi^{j}-g^{i p} \tilde{\partial}^{r} \partial_{p} \partial_{j} \xi^{j}, \\
\delta_{\xi}^{(0)}\left(\partial_{i} \tilde{\partial}^{k} b_{j k}\right)= & \mathcal{L}_{\xi}\left(\partial_{i} \tilde{\partial}^{k} b_{j k}\right)+\partial_{i} \partial_{k} \xi^{p} \tilde{\partial}^{k} b_{j p}+\partial_{k} \xi^{p} \partial_{i} \tilde{\partial}^{k} b_{j p}+\partial_{i} \partial_{j} \xi^{p} \tilde{\partial}^{k} b_{p k} \\
& +\partial_{i} \tilde{\partial}^{k} \xi^{p} \partial_{p} b_{j k}+\tilde{\partial}^{k} \xi^{p} \partial_{i} \partial_{p} b_{j k}+\partial_{i} \tilde{\partial}^{k} \partial_{j} \xi^{p} b_{p k}-\tilde{\partial}^{k} \partial_{j} \xi^{p} \partial_{i} b_{k p} .
\end{aligned}
$$

Next, we look at $\delta^{(1)}$. It acts trivially on $d$, while on $g$ and $b$ we find the non-linear transformations

$$
\begin{aligned}
\delta_{\xi}^{(1)} g_{i j} & =2\left(\tilde{\partial}^{k} \xi^{l}-\tilde{\partial}^{l} \xi^{k}\right) g_{k(i} b_{j) l} \\
\delta_{\xi}^{(1)} g^{i j} & =-\left(\tilde{\partial}^{i} \xi^{k}-\tilde{\partial}^{k} \xi^{i}\right) g^{j l} b_{l k}+(i \leftrightarrow j), \\
\delta_{\xi}^{(1)} b_{i j} & =g_{i k}\left(\tilde{\partial}^{l} \xi^{k}-\tilde{\partial}^{k} \xi^{l}\right) g_{l j}+b_{i k}\left(\tilde{\partial}^{l} \xi^{k}-\tilde{\partial}^{k} \xi^{l}\right) b_{l j} .
\end{aligned}
$$

The variation $\delta^{(1)} \mathcal{L}^{(0)}$ is lengthy because we have to vary the metric everywhere, not only under partial derivatives. For this it is convenient to slightly rewrite $\mathcal{L}^{(0)}$ with less appearances of metrics and inverse metrics,

$$
\mathcal{L}^{(0)}=e^{-2 d}\left(\frac{1}{4} g^{p q} \partial_{p} g^{i j} \partial_{q} g_{i j}-\frac{1}{2} g^{i j} \partial_{j} g^{k l} \partial_{l} g_{i k}-\partial_{i} \partial_{j} g^{i j}+4 g^{i j} \partial_{i} d \partial_{j} d-\frac{1}{12} g^{i l} g^{j p} g^{k q} H_{i j k} H_{l p q}\right) .
$$

Here we need to vary everything under $\delta^{(1)}$ as given in $(\overline{B .23})-(\overline{B .25})$. Then we have to vary all terms involving partial derivatives in (B.5) according to (B.1) and (B.16) $-(\underline{B} .22)$. A tedious but straightforward calculation then shows that these two sets of variations precisely cancel, thus proving gauge invariance.

\section{Properties of the curvature scalar}

In section $\$ 4.2$ we defined a curvature scalar $\mathcal{R}$ and claimed that it transforms as (4.21), and that the action (4.22) is equivalent to (2.54). We also claimed that the dilaton equation of motion is precisely $\mathcal{R}=0$. In this appendix we verify these claims. 
We first test these ideas for $S^{(0)}$. We add a total derivative to the corresponding Lagrangian density $\mathcal{L}^{(0)}$ in (3.18), so that the dilaton equation arises just by varying the exponential prefactor. Then we confirm that the resulting Lagrangian density $\mathcal{L}^{\prime(0)}$ agrees with $\mathcal{R}$, also evaluated to leading order in the tilde-derivative expansion. Indeed, choosing the total derivative term

$$
\mathcal{L}^{\prime(0)} \equiv \mathcal{L}^{(0)}+\partial_{i}\left[e^{-2 d} g^{i j}\left(4 \partial_{j} d+\partial^{k} g_{j k}\right)\right]
$$

leads to

$$
\begin{aligned}
\mathcal{L}^{(0)}=e^{-2 d}[ & -\frac{1}{4} g^{i k} g^{j l} \partial^{p} g_{k l} \partial_{p} g_{i j}+\frac{1}{2} g^{k l} \partial^{j} g_{i k} \partial^{i} g_{j l}+\partial_{i}\left(g^{i j} \partial^{k} g_{j k}\right)-\frac{1}{12} H^{2} \\
& \left.+4\left(-\partial^{i} d \partial^{j} g_{i j}-\partial^{i} d \partial_{i} d+g^{i j} \partial_{i} \partial_{j} d\right)\right] .
\end{aligned}
$$

It is straighforward to confirm that the variation of $d$ in the terms on the last line of the above equation yields a total derivative. This confirms that the dilaton equation of motion that results from $\mathcal{L}^{\prime(0)}$ is obtained by varying only the dilaton exponential; the equation of motion is simply the vanishing of the terms within square brackets.

It is natural to expect that $\mathcal{L}^{(0)}$ is the Lagrangian associated with $S^{\prime}$, when tilde-derivatives are set to zero. To confirm this we evaluate $\mathcal{R}$ for $\tilde{\partial}=0$. A computation starting with (4.33) gives

$$
\begin{aligned}
\left.\mathcal{R}\right|_{\tilde{\partial}=0}= & 2\left(g^{i p}\left[\partial_{p}-\frac{1}{2} \partial^{k} \mathcal{E}_{k p}\right] \partial_{i} d+g^{i p}\left[\partial_{p}-\frac{1}{2} \partial^{k} \mathcal{E}_{p k}\right] \partial_{i} d\right) \\
& +\frac{1}{2}\left(g^{i p}\left[\partial_{p}-\frac{1}{2} \partial^{k} \mathcal{E}_{k p}\right] \partial^{j} \mathcal{E}_{i j}+g^{i p}\left[\partial_{p}-\frac{1}{2} \partial^{k} \mathcal{E}_{p k}\right] \partial^{j} \mathcal{E}_{j i}\right) \\
& +\frac{1}{4} g^{i j}\left(\partial^{k} \mathcal{E}_{l j} \partial^{l} \mathcal{E}_{k i}+\partial^{k} \mathcal{E}_{j l} \partial^{l} \mathcal{E}_{i k}\right)-\frac{1}{4} g^{i j}\left(\partial^{l} \mathcal{E}_{l j} \partial^{k} \mathcal{E}_{k i}+\partial^{l} \mathcal{E}_{j l} \partial^{k} \mathcal{E}_{i k}\right) \\
& -\frac{1}{4} g^{i k} g^{j l} \partial^{p} \mathcal{E}_{k l} \partial_{p} \mathcal{E}_{i j}-2 \partial^{j} g_{i j} \partial^{i} d-4 \partial^{i} d \partial_{i} d .
\end{aligned}
$$

Collecting terms this becomes

$$
\begin{aligned}
\left.\mathcal{R}\right|_{\tilde{\partial}=0}= & 4\left(-\partial^{i} d \partial^{j} g_{i j}-\partial^{i} d \partial_{i} d+g^{i j} \partial_{i} \partial_{j} d\right) \\
& +\frac{1}{4} g^{i j}\left(\partial^{k} \mathcal{E}_{l j} \partial^{l} \mathcal{E}_{k i}+\partial^{k} \mathcal{E}_{j l} \partial^{l} \mathcal{E}_{i k}\right)-\frac{1}{4} g^{i j}\left(\partial^{l} \mathcal{E}_{l j} \partial^{k} \mathcal{E}_{k i}+\partial^{l} \mathcal{E}_{j l} \partial^{k} \mathcal{E}_{i k}\right) \\
& -\frac{1}{4} g^{i j}\left(\partial^{k} \mathcal{E}_{k j} \partial^{l} \mathcal{E}_{i l}+\partial^{k} \mathcal{E}_{j k} \partial^{l} \mathcal{E}_{l i}\right)-\frac{1}{4} g^{i k} g^{j l} \partial^{p} \mathcal{E}_{k l} \partial_{p} \mathcal{E}_{i j}+\partial^{i} \partial^{j} g_{i j} .
\end{aligned}
$$

A short calculation then shows that, as expected,

$$
\mathcal{L}^{\prime(0)}=\left.e^{-2 d} \mathcal{R}\right|_{\tilde{\partial}=0} .
$$

So far we have proved that $e^{-2 d} \mathcal{R}$ and the original Lagrangian $\mathcal{L}$ differ only by a total derivative when restricted to $\tilde{\partial}=0$. Indeed, combining (C.27) and (C.31) we get

$$
\left.e^{-2 d} \mathcal{R}\right|_{\tilde{\partial}=0}=\left.\mathcal{L}\right|_{\tilde{\partial}=0}+\partial_{i}\left[e^{-2 d} g^{i j}\left(4 \partial_{j} d+\partial^{k} g_{j k}\right)\right] .
$$


We now state a simple but useful lemma. Given two $O(D, D)$ scalars $A(x, \tilde{x})$ and $B(x, \tilde{x})$ that differ by total derivative terms, then after an $O(D, D)$ transformation they again differ by total derivative terms. The proof is immediate. Let the scalars $A, B$ differ by total derivative terms in the $(x, \tilde{x})$ frame,

$$
A(x, \tilde{x})=B(x, \tilde{x})+\partial_{i} F^{i}+\tilde{\partial}^{i} \tilde{F}_{i} .
$$

Being $O(D, D)$ scalars we have $A(x, \tilde{x})=A^{\prime}\left(x^{\prime}, \tilde{x}^{\prime}\right)$ and $B(x, \tilde{x})=B^{\prime}\left(x^{\prime}, \tilde{x}^{\prime}\right)$, so that the above becomes

$$
A^{\prime}\left(x^{\prime}, \tilde{x}^{\prime}\right)=B^{\prime}\left(x^{\prime}, \tilde{x}^{\prime}\right)+\partial_{i} F^{i}+\tilde{\partial}^{i} \tilde{F}_{i}
$$

Recall now that under an $O(D, D)$ transformation (2.56) the derivatives $\partial^{M}=\left(\partial_{i}, \tilde{\partial}^{i}\right)$ transform as

$$
\left(\begin{array}{c}
\partial^{\prime} \\
\tilde{\partial}^{\prime}
\end{array}\right)=\left(\begin{array}{ll}
a & b \\
c & d
\end{array}\right)\left(\begin{array}{l}
\partial \\
\tilde{\partial}
\end{array}\right) \rightarrow\left(\begin{array}{l}
\partial \\
\tilde{\partial}
\end{array}\right)=\left(\begin{array}{ll}
d^{t} & b^{t} \\
c^{t} & a^{t}
\end{array}\right)\left(\begin{array}{l}
\partial^{\prime} \\
\tilde{\partial}^{\prime}
\end{array}\right)
$$

Since $a, b, c, d$ are constant matrices, the total derivative terms in (C.34) remain total derivatives in the primed variables. This proves the lemma.

Returning to our application, consider the two $O(D, D)$ scalars: $\mathcal{L}$ and $e^{-2 d} \mathcal{R}$. Given the strong constraint, one can always use an $O(D, D)$ transformation to rotate into a frame where fields have no $\tilde{x}$ dependence, allowing us to set $\tilde{\partial}=0$. In this frame we have verified that the two scalars differ by a total derivative. The lemma implies that the original Lagrangians $\mathcal{L}$ and $e^{-2 d} \mathcal{R}$ differed by a total derivative before the $O(D, D)$ transformation.

We can describe the general form of the total derivatives more explicitly by writing

$$
e^{-2 d} \mathcal{R}=\mathcal{L}+\partial_{M} \Theta^{M}
$$

The last term on the right-hand side must be an $O(D, D)$ scalar since the other two terms are. This happens if $\Theta^{M}=\left(\tilde{\theta}_{i}, \theta^{i}\right)$ transforms in the fundamental of $O(D, D)$. The components of $\Theta^{M}$ can be constructed as

$$
\theta^{i}=\frac{1}{2}\left(-Y^{i}+\bar{Y}^{i}\right), \quad \tilde{\theta}_{i}=\frac{1}{2}\left(\mathcal{E}_{j i} Y^{j}+\mathcal{E}_{i j} \bar{Y}^{j}\right)
$$

where $Y$ and $\bar{Y}$, to be determined below, transform with $M$ and $\bar{M}$ and are therefore $O(D, D)$ tensors in the sense of 82.4 . This ensures that $\Theta^{M}$ transforms in the fundamental of $O(D, D)$. This statement should be compared with equations (2.30) that relate $\partial_{M}=\left(\tilde{\partial}^{i}, \partial_{i}\right)$ transforming in the fundamental to $\mathcal{D}$ and $\overline{\mathcal{D}}$ transforming with $M$ and $\bar{M}$, respectively. For these variables the proof was given in $\S 4.2$ of [2], and this proof readily extends to any $\Theta^{M}$ defined as in (C.37). Specifically, here we have

$$
\begin{aligned}
& Y^{i}=-e^{-2 d} g^{i j}\left(4 \mathcal{D}_{j} d+g^{k l} \overline{\mathcal{D}}_{k} \mathcal{E}_{j l}\right), \\
& \bar{Y}^{i}=e^{-2 d} g^{i j}\left(4 \overline{\mathcal{D}}_{j} d+g^{k l} \mathcal{D}_{k} \mathcal{E}_{l j}\right) .
\end{aligned}
$$

These expressions are fixed by the requirement that they transform covariantly under $O(D, D)$, i.e., with $M$ and $\bar{M}$, and that $\theta^{i}$ correctly reduces to (C.27) for $\tilde{\partial}=0$. As a consistency check one may verify that $\tilde{\theta}_{i}$ reduces for $\partial=0$ to the T-dual expression, the one which is obtained from (C.27) by mapping $\mathcal{E} \rightarrow \tilde{\mathcal{E}}, \partial \rightarrow \tilde{\partial}$ as in $\$ 3.2$. 
We now relate $\mathcal{L}^{\prime(0)}$ or $\left.\mathcal{R}\right|_{\tilde{\partial}=0}$ to the standard quantities in the conventional action. A calculation similar to that in $\$ 3.1$ gives

$$
\left.\mathcal{R}\right|_{\tilde{\partial}=0}=R+4 \square \phi-4(\partial \phi)^{2}-\frac{1}{12} H^{2}
$$

This means that to zeroth-order in the tilde derivative expansion we have

$$
S_{*}^{\prime}=\int d x \sqrt{-g} e^{-2 \phi}\left[R+4 \square \phi-4(\partial \phi)^{2}-\frac{1}{12} H^{2}\right] .
$$

Equation (C.40) also gives further evidence that $\mathcal{R}$ is a scalar under gauge transformations. We use an $O(D, D)$ transformation to fields with no $\tilde{x}$ dependence so that $\mathcal{R}$ takes the above form, and the gauge transformations become the familiar ones for which the right-hand side is clearly a scalar.

\section{References}

[1] T. Kugo and B. Zwiebach, "Target space duality as a symmetry of string field theory," Prog. Theor. Phys. 87, 801 (1992) arXiv:hep-th/9201040].

[2] C. Hull and B. Zwiebach, "Double Field Theory," JHEP 0909 (2009) 099 arXiv:0904.4664 [hepth]].

[3] C. Hull and B. Zwiebach, "The gauge algebra of double field theory and Courant brackets," JHEP 0909 (2009) 090 arXiv:0908.1792 [hep-th]].

[4] A. A. Tseytlin, "Duality Symmetric Formulation Of String World Sheet Dynamics," Phys. Lett. B 242, 163 (1990); "Duality Symmetric Closed String Theory And Interacting Chiral Scalars," Nucl. Phys. B 350, 395 (1991).

[5] W. Siegel, "Superspace duality in low-energy superstrings," Phys. Rev. D 48, 2826 (1993) arXiv:hep-th/9305073; ; "Two vierbein formalism for string inspired axionic gravity," Phys. Rev. D 47, 5453 (1993) arXiv:hep-th/9302036.

[6] B. Zwiebach, "Closed string field theory: Quantum action and the B-V master equation," Nucl. Phys. B 390, 33 (1993) arXiv:hep-th/9206084.

[7] T. Courant, "Dirac Manifolds." Trans. Amer. Math. Soc. 319: 631-661, 1990.

[8] N. Hitchin, "Generalized Calabi-Yau manifolds," Q. J. Math. 54 (2003), no. 3, 281-308, arXiv:math.DG/0209099.

[9] M. Gualtieri, "Generalized complex geometry," PhD Thesis (2004). arXiv:math/0401221v1 [math.DG]

[10] Z.-J. Liu, A. Weinstein, and P. Xu, "Manin triples for Lie bialgebroids," J. Diff. Geom., 45: 547-574, 1997. arXiv:dg-ga/9508013 
[11] M. Gualtieri, "Branes on Poisson varieties," arXiv:0710.2719v1 [math.DG].

[12] A. Giveon, E. Rabinovici and G. Veneziano, "Duality in String Background Space," Nucl. Phys. B 322 (1989) 167.

[13] A. D. Shapere and F. Wilczek, "Selfdual Models with Theta Terms," Nucl. Phys. B 320 (1989) 669.

[14] A. Giveon, M. Porrati and E. Rabinovici, "Target space duality in string theory," Phys. Rept. 244 (1994) 77 arXiv:hep-th/9401139].

[15] Y. Michishita, "Field redefinitions, T-duality and solutions in closed string field theories," JHEP 0609, 001 (2006) arXiv:hep-th/0602251.

[16] T. Damour, S. Deser and J. G. McCarthy, "Nonsymmetric Gravity Theories: Inconsistencies And A Cure," Phys. Rev. D 47 (1993) 1541 arXiv:gr-qc/9207003.

[17] A. A. Tseytlin, "Sigma model approach to string theory," Int. J. Mod. Phys. A 4, 1257 (1989).

[18] J. Polchinski, "String theory. Vol. 1: An introduction to the bosonic string," Cambridge, UK: Univ. Pr. (1998).

[19] C. M. Hull and R. A. Reid-Edwards, "Gauge Symmetry, T-Duality and Doubled Geometry," JHEP 0808 (2008) 043 arXiv:0711.4818 [hep-th]].

[20] C. M. Hull and R. A. Reid-Edwards, "Non-geometric backgrounds, doubled geometry and generalised T-duality," JHEP 0909 (2009) 014 [arXiv:0902.4032 [hep-th]].

[21] C. M. Hull, "A geometry for non-geometric string backgrounds," JHEP 0510 (2005) 065 arXiv:hep-th/0406102.

[22] C. M. Hull, "Doubled geometry and T-folds," JHEP 0707 (2007) 080 arXiv:hep-th/0605149.

[23] C. M. Hull, "Generalised geometry for M-theory,” JHEP 0707 (2007) 079 arXiv:hep-th/0701203.

[24] P. P. Pacheco and D. Waldram, "M-theory, exceptional generalised geometry and superpotentials," JHEP 0809 (2008) 123 [arXiv:0804.1362 [hep-th]].

[25] J. Maharana and J. H. Schwarz, "Noncompact symmetries in string theory," Nucl. Phys. B 390 (1993) 3 arXiv:hep-th/9207016.

[26] E. Cremmer and B. Julia, "The SO(8) Supergravity," Nucl. Phys. B 159 (1979) 141.

[27] B. de Wit and H. Nicolai, "Hidden symmetries, central charges and all that," Class. Quant. Grav. 18 (2001) 3095 arXiv:hep-th/0011239.

[28] C. C. Chevalley, "The Algebraic Theory of Spinors," Columbia University Press, 1954. 
[29] E. Artin, "Geometric Algebra," Interscience Publishers, Inc., 1957.

[30] T. H. Buscher, "A Symmetry of the String Background Field Equations," Phys. Lett. B 194 (1987) 59, "Path Integral Derivation of Quantum Duality in Nonlinear Sigma Models," Phys. Lett. B 201 (1988) 466.

[31] A. Giveon and M. Rocek, "Generalized duality in curved string backgrounds," Nucl. Phys. B 380 (1992) 128 arXiv:hep-th/9112070.

[32] C. M. Hull and P. K. Townsend, "Unity of superstring dualities," Nucl. Phys. B 438 (1995) 109 arXiv:hep-th/9410167.

[33] C. Hillmann, "Generalized E(7(7)) coset dynamics and D=11 supergravity," JHEP 0903 (2009) 135 arXiv:0901.1581 [hep-th]]. 